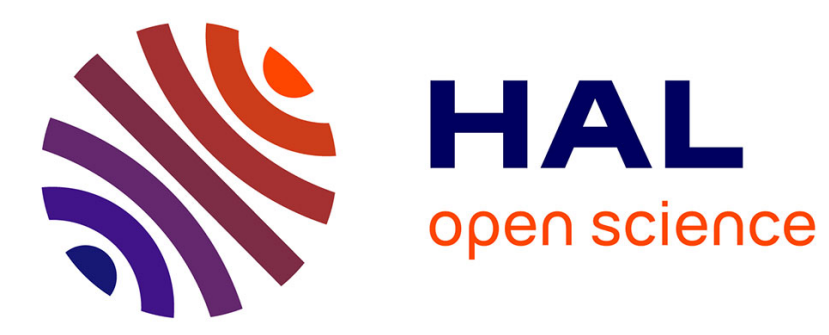

\title{
Corps et objets interdits dans les sanctuaires (monde grec, Ve av. - IIIe ap. J.-C.)
}

Marie Augier

\section{To cite this version:}

Marie Augier. Corps et objets interdits dans les sanctuaires (monde grec, Ve av. - IIIe ap. J.-C.).

Archimède: archéologie et histoire ancienne, 2020, 7, pp.6-22. halshs-02892823

\section{HAL Id: halshs-02892823 \\ https://shs.hal.science/halshs-02892823}

Submitted on 7 Jul 2020

HAL is a multi-disciplinary open access archive for the deposit and dissemination of scientific research documents, whether they are published or not. The documents may come from teaching and research institutions in France or abroad, or from public or private research centers.
L'archive ouverte pluridisciplinaire HAL, est destinée au dépôt et à la diffusion de documents scientifiques de niveau recherche, publiés ou non, émanant des établissements d'enseignement et de recherche français ou étrangers, des laboratoires publics ou privés. 


\section{ARCHIMĖDE N N 7}

DOSSIER THÉMATIQUE :

GESTES RITUELS. DE LA TRACE À L'INTERPRÉTATION

1 Sylvie DONNAT, Jean-Marie HUSSER

Le rite et ses traces. Perspectives méthodologiques

6 Marie AUGIER

Corps et objets interdits dans les sanctuaires (monde grec, ve av. - III ${ }^{\mathrm{e}}$ ap. J.-C.)

23 Claire CAMBERLEIN

Offrir l'antique à la divinité. L'exemple de l'adyton du sanctuaire de Vryokastro sur l'île de Kythnos (Cyclades)

37 Sylvie DONNAT

Les gestes rituels autour des papyrus-amulettes (Égypte, fin du II millénaire av. n. è.)

51 Jean-Marie HUSSER

Entre rituel, mémorial et littérature de propagande. À propos de deux textes ougaritiques au genre mal défini

62 Anne JACQUEMIN

Rituels efficaces pour fonder ou déplacer un culte en Grèce ancienne

76 Françoise LAROCHE-TRAUNECKER

Les rituels de construction des temples égyptiens. Gestes représentés sur les parois et actes attestés par des fouilles à Karnak

96 Denis MONNERIE

Circulation des objets et élaboration des relations dans les cérémonies du nord de la Kanaky Nouvelle-Calédonie

\section{ACTUALITÉ DE LA RECHERCHE}

294 VARIA 


\title{
CORPS ET OBJETS INTERDITS DANS LES SANCTUAIRES (MONDE GREC, VE AV. - IIIE AP. J.-C.)
}

\author{
Marie AUGIER \\ Docteure en histoire grecque \\ Université de Strasbourg \\ UMR 7044 Archimède \\ marie.augier@gmail.com
}

\section{RÉSUMÉ}

L'article se propose de revenir sur ce que les prescriptions cathartiques, ces inscriptions placées devant l'entrée des sanctuaires et limitant leur accès, indiquent des gestes rituels qui y étaient pratiqués, tels les gestes purificatoires. Ces documents épigraphiques précisent les actions ou les situations vécues hors du sanctuaire qui entraînaient une impureté rituelle et interdisent la consommation préalable de certains aliments ou le port de certains types de vêtement et objets par souci d'eukosmia. Ils marquent ainsi la limite entre deux espaces, l'espace sacré et l'espace extérieur au sanctuaire. Ils mettent donc au jour en creux les actes qui ne

\section{MOTS-CLÉS}

Grèce antique épigraphie,

rituels,

pureté/purification,

souillure,

corps,

aliments,

vêtements. pouvaient s'accomplir dans l'espace sacré, les corps qui ne pouvaient y pénétrer ou les vêtements et objets qui ne pouvaient $s^{\prime} y$ trouver à moins qu'ils ne soient des anathemata.
This article analyses the light that cathartic prescriptions -these inscriptions placed at the temples gate to regulate their access- shed on ritual gestures performed in the sanctuaries, such as purification. These epigraphic texts specify which actions and circumstances experienced outside the temple brought ritual impurity; they also prohibit the consumption, prior to entering the temple, of some food, as well as wearing specific clothes and objects, for the sake of eukosmia. As such, cathartic prescriptions indicate the threshold between two spaces, the sacred one, and the other outside the sanctuary. By so doing, they implicitly highlight actions that could not be performed in the sacred space, the bodies that could not enter it, and the clothes and objects that could not be in it, unless they were anathemata.

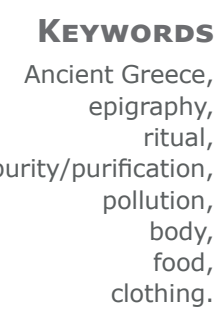


Le sanctuaire est un espace à part, réservé à la divinité, le lieu où des rituels en son honneur sont accomplis. Aussi l'accès à cet espace est-il limité et encadré. Les prescriptions cathartiques, ces inscriptions placées devant l'entrée des sanctuaires indiquent les « corps » et les objets qui n'avaient pas leur place dans le sanctuaire, soit parce qu'ils apportaient avec eux une souillure rituelle, soit parce qu'ils ne seyaient pas à la divinité. Cette documentation qui s'échelonne du ve siècle av. notre ère au III ${ }^{\mathrm{e}}$ siècle de notre ère et qui regroupe une quarantaine d'inscriptions de toutes origines dans le monde grec précise quels individus étaient impurs - en raison du contact avec un mort, de la naissance ou des relations sexuelles par exemple -, mais aussi ce qu'il ne fallait pas porter sur soi ou apporter avec soi - vêtements, parures ou objets divers. Ces inscriptions concernent des personnes et des objets dont, en vertu d'un interdit, on ne devrait pas trouver la trace dans un sanctuaire. Connaître ces interdits est donc important pour aborder les gestes du rituel, car ils permettent de mettre au jour ce qui ne pouvait s'y trouver ni s'y accomplir. Face à ces interdits rituels plusieurs questions se posent : de quelle nature les impuretés corporelles sont-elles ? Comment peut-on s'en débarrasser ? Ces impuretés touchent-elle à la nature de l'individu ? Et que nous indiquent-elles alors de la limite entre les deux lieux : I'espace sacré et l'espace profane ? Enfin quelles autres pratiques interdites dans les sanctuaires suggèrent-elles ? Qu'est-ce qui motivait le rejet de certains objets ou vêtements ou parures du sanctuaire? Trouve-t-on néanmoins trace de ces objets interdits?

Ces inscriptions, placées à l'entrée des sanctuaires, exposaient les règles de pureté et constituent pour nous des traces de gestes rituels liés à des pratiques de purification lorsqu'elles paraissent associées à des bassins de lustration retrouvés in situ.

Après une brève présentation du corpus et de ses caractéristiques, l'étude se propose de présenter ces deux types d'interdits : ceux qui touchent les personnes qui ne pouvaient accéder au sanctuaire et les actions qui y étaient prohibées (accoucher, mourir, allaiter, avoir des relations sexuelles...), mais aussi ceux qui ont trait à des objets qui laissent ainsi des traces en négatif dans les sanctuaires (on interdit le port de certains vêtements ou objets dans le sanctuaire), ou qui, s'ils s'y trouvent, peuvent être le résultat d'une transgression.

\section{UN CORPUS DISPARATE ?}

Le corpus des prescriptions cathartiques regroupe une quarantaine d'inscriptions [1] plus ou moins bien conservées. Ces inscriptions ont été regroupées dans deux tableaux placés dans les annexes à la fin de cet article. Le premier précise pour chaque inscription du corpus quel(s) interdit(s) rituel(s) y étai(en)t indiqué(s), tandis que le second détaille les animaux, vêtements et objets rejetés du sanctuaire dans certaines des inscriptions. La lecture de ces tableaux permet de constater que l'ambitus chronologique est fort large, car les documents s'échelonnent du ve siècle av. notre ère au $\mathrm{III}^{\mathrm{e}}$ siècle de notre ère, avec une majorité de textes à partir de la période hellénistique. Cela ne veut pas dire que des prescriptions cathartiques n'étaient pas édictées ou l'étaient moins aux périodes archaïque et classique, mais simplement que celles-ci ne nous sont parvenues qu'en petit nombre [2]. Elles ont pu être perdues ou encore regravées comme le prouve un

[1] Les références des 40 inscriptions seront données avec les abréviations usuelles; je choisis néanmoins pour les inscriptions éditées dans les trois volumes de $F$. Sokolowski les abréviations suivantes : pour des inscriptions tirées des Lois sacrées des cités grecques, 'LSCG' ; pour les Lois sacrées des cités grecques, Supplément, 'LSS' ; pour les Lois sacrées d'Asie Mineure, 'LSAM'. Plusieurs références séparées par le signe ' ' pourront être données pour une même inscription ; quand les inscriptions ont été publiées dans le CGRN [Collection of Greek Ritual Norms] - ce qui n'est pas le cas de toutes les inscriptions du corpus -, la référence CGRN sera ainsi également indiquée.

[2] Sept inscriptions du corpus sont datées du ve ou du IV siècle av. notre ère : LSCG 97/ CGRN 35, LSS 115/ CGRN 99, LSAM 29/ CGRN 71, LSS 31/ CGRN 65, LSS 32, LSS 63/ CGRN 27 et Pouilloux, Rech I, n 155. 
des règlements du corpus en provenance de Délos et daté de 116/115 av. J.-C. [3] Il précise que le prêtre a fait remplacer, lors de son entrée en fonction, la stèle brisée dans le sanctuaire de Zeus Kynthios et Athéna Kynthia :

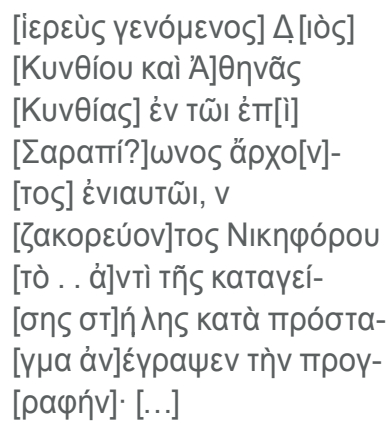

Si l'inscription ne permet pas de dater l'origine des prescriptions qu'elle contient, elle est révélatrice du fait que la date d'une inscription ne correspond pas forcément à la date d'instauration des prescriptions qui peuvent être bien plus anciennes.

Le cadre géographique est tout aussi large, puisque les inscriptions proviennent à la fois de Grèce continentale [4], mais aussi d'Asie Mineure [5], des îles de la mer Égée [6] ou encore d'Égypte [7] ou de

\section{[3] LSS 59.}

[4] C'est par exemple le cas des inscriptions LSS 31/ CGRN 65, Tégée ; SEG XXVIII 421/LuPU 2005, n 7/ CGRN 155, Mégalopolis ; LSCG 55, Sounion.

[5] Par exemple : LSAM 29/CGRN 71, Métropolis, Ionie ; LSAM 18/ CGRN 211, Méonie, Lydie ; LSAM 12/ CGRN 212, Pergame ; LSAM 20/ CGRN 191, Philadelphie, Lydie ; LSAM 51/ CGRN 214, Milet ; LSAM 14, Pergame.

[6] Par exemple : LSCG 151/ CGRN 86, Cos ; LSCG 156/ CGRN 85, Cos ; LSCG 124/ CGRN 181, Erésos, Lesbos ; LSCG 171/ CGRN 162, LSS 54/ CGRN 217 Délos ; LSCG 95/ CGRN 203, Délos ; LSS 59, Délos ; LSS 108, Rhodes ; LSCG 139, Lindos, Rhodes ; LSS 91, Lindos, Rhodes.

[7] LSS 119/ CGRN 144, Ptolémaïs.

[8] LSS 115/ CGRN 99.

[9] Sur l'impureté corporelle : WÄCHTER 1910, Moulinier 1950, PARKer 1996, BendLin 2007.

[10] Voir infra.

[11] LSS 119/ CGRN 144.

[12] Voir infra.

[13] Voir infra. Il s'agit des mots de la famille de סıa$Ө$ opá, terme qui peut être compris comme avortement ou fausse couche.

[14] LSS 91. Sur l'allaitement, BodIou 2011.
Cyrénaïque [8]. La grande disparité géographique des documents ne se retrouve pas dans les prescriptions qu'ils contiennent, mais on note au contraire une relative homogénéité dans les conditions d'accès aux sanctuaires et les causes de souillure. Les souillures [9] les plus courantes mentionnées dans notre corpus, en dehors des prescriptions propres à chaque sanctuaire, étaient liées au contact avec le cadavre [10] ou le malade [11], aux relations sexuelles [12], à l'accouchement, à l'avortement ou à la fausse couche [13], et plus rarement à I'allaitement [14], aux menstruations [15] ou à l'exposition d'un enfant [16]. La consommation de certaines denrées [17] était parfois également interdite et on interdisait enfin l'accès au sanctuaire à des personnes portant certaines couleurs ou matières comme le cuir [18] ou certaines coiffures [19], mais aussi des bijoux [20] ou des armes [21].

Il est en outre assez remarquable de noter que pour certains types de prescriptions, on retrouve les mêmes termes ou les mêmes expressions pour qualifier une impureté rituelle dans des inscriptions de périodes et de lieux fort éloignés. C'est ainsi le cas des interdits rituels découlant d'une relation sexuelle : l'expression ámò yuvaıkós est employée dans douze prescriptions [22] ; pour les funérailles, on retrouve neuf

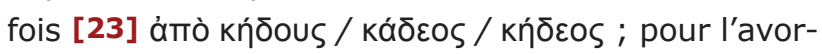
tement ou la fausse couche, ce sont des mots de la famille de ঠıаழӨopá [24], un terme au sens ambiguë, qui sont utilisés à huit reprises, tandis que pour l'accouchement, on utilise soit le substantif $\lambda \varepsilon ́$ Xos [25],

[15] SEG XXVIII 421/ LUPU 2005 n० 7/ CGRN 155 ; LSS 54/ CGRN 217 ; LSCG 55; LSS 91 ; DECOURT \& TZIAPHALIAS 2015.

[16] LSS 119/ CGRN 144 ; LSAM 20/ CGRN 191.

[17] SEG XXVIII 421/ LUPU 2005 n० 7/ CGRN 155 ; LSS 54/ CGRN 217 ; LSCG 95/ CGRN 203 ; LSS 108 ; LSCG 139 ; LSCG 55 ; DECOURT \& TZIAPHALIAS 2015.

[18] L'interdiction de porter des vêtements en cuir est à mettre en relation avec la souillure issue du contact avec un mort dans la mesure où le cuir est de la peau d'une bête morte.

[19] LSCG 124/ CGRN 181 ; LSS 59 ; LSS 91 ; LSAM 14. [20] LSCG 124/ CGRN 181 ; LSAM 14.

[21] LSCG 124/ CGRN 181 ; LSS 59 ; LSS 91.

[22] LSAM 12/ CGRN 212, LSAM 14, LSAM 18/ CGRN 211, LSAM 29 / CGRN 71, LSAM 51/ CGRN 214 ; LSS 54/ CGRN 217, LSS 59, LSS 115/ CGRN 99 et LSS 119/ CGRN 144 ; LSCG 55, LSCG 95/ CGRN 203, LSCG 124/ CGRN 181.

[23] LSAM 12/ CGRN 212, LSAM 14, LSAM 18/ CGRN 211, LSAM 29 / CGRN 71, LSAM 51/ CGRN 214; LSCG 55, LSCG 124/ CGRN 181 ; LSCG 139 ; LSS 91.

[24] LUPU $2005 n^{\circ} 7 /$ CGRN 155 ; LSS 54/ CGRN 217, LSS 91 ; LSCG 55, LSCG 124/ CGRN 181, LSCG 139 et LSCG 171/ CGRN 162 ; DECOURT \& TZIAPHALIAS 2015. [25] LUPU $2005 n^{\circ} 7 /$ CGRN 155 ; LSCG 171/ CGRN 162 ; LSS 91 et LSS 115/ CGRN 99 ; DECOURT \& TZIAPHALIAS 2015. 
soit la forme verbale тікт $\omega$ [26]. Enfin, pour certaines prescriptions, comme pour l'avortement, le délai pour recouvrer sa pureté rituelle est toujours autour d'une quarantaine de jours. L'emploi de ce vocabulaire formulaire est d'autant plus significatif que, lorsqu'il s'agit de prescriptions moins fréquentes ou marginales, les termes employés sont beaucoup plus variés. Les menstruations ne sont évoquées que dans de rares prescriptions et, lorsque c'est le cas, la manière de les désigner est bien moins stéréotypée que pour d'autres interdits plus coutumiers du monde grec [27].

Cette cohérence dans les causes de souillure et dans la désignation de certaines d'entre elles se retrouve alors même que les prescriptions règlementent l'accès à des sanctuaires dédiés à des divinités différentes [28], qu'elles soient d'origine grecque (Apollon [29] ; Artémis [30] ; Artémis et Zeus Hikésios [31] ; Athéna Niképhoros [32] ; Zeus Kynthios et Athéna Kynthia [33] ; Artémis Kithônè [34] ; Artémis Phylakè [35] ; Athéna [36] ; Asclépios [37] ; Zeus Polieus [38]...) ou étrangère (Déesse-Mère [39] ; Isis, Sarapis et Anoubis [40] ; Dieux Syriens [41] ; Mên [42]...).

Le corpus se compose ainsi de documents d'origines et de dates variées qui étaient placés devant des sanctuaires honorant des divinités tout aussi variées ; malgré cette disparité, on note une similarité dans certaines impuretés et certains interdits, ce qui n'est pas contradictoire avec l'ajout de spécificités propres au lieu, à la période ou au culte rendu dans l'espace sacré. Ainsi, constate-t-on dans cinq règlements du corpus I'ajout de sentences que I'on pourrait qualifier de «morales ». D'après ces inscriptions, l'impureté entachait non seulement le corps, mais aussi

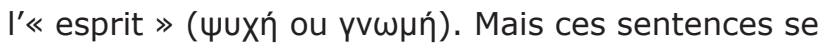
trouvent dans des inscriptions qui se démarquent par leur sévérité, par leur date récente et/ou par l'origine non grecque du culte concerné [43]. Il est d'ailleurs assez difficile de comprendre de quelle nature était la souillure. Si Antiphon par exemple [44] emploie le substantif $\mu$ iaøua dans un passage pour désigner une souillure rituelle, ce terme n'est pas employé dans la documentation épigraphique. Il existe en revanche en grec un vocabulaire varié [45] qui ne nous permet pas de proposer une définition claire et uniforme de ce que serait une souillure rituelle ou une impureté corporelle dans un contexte religieux. L'adjectif kaӨapós ou le verbe $\kappa \alpha \theta \alpha \rho i \zeta \omega$ se trouvera ainsi dans certains règlements du corpus, mais de manière générale la formulation elliptique des inscriptions ne permet pas de préciser ce que les Grecs entendaient par « souillure » [46] : une impureté corporelle certes, mais qui

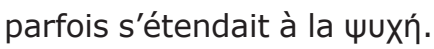

On ne donne en effet généralement que deux informations dans ces listes : l'impureté rituelle et la manière de l'effacer, généralement une durée (d'un à
[26] LSCG 124/ CGRN 181 ; LSAM 12/ CGRN 212, LSAM 14, LSAM 51/ CGRN 214 et LSAM 119.

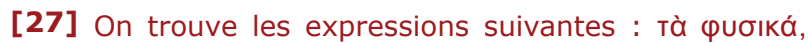

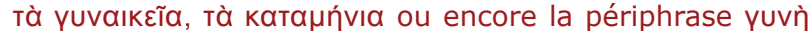
$\sigma \mu \eta \sigma \alpha \mu \varepsilon ́ v \eta$. Nous pouvons donc en déduire que cet interdit n'est pas d'origine grecque ; sur cette question, AUgIER 2017b.

[28]Le nombre d'inscriptions ne permet malheureusement pas de comparer les comportements rituels pour chaque divinité.

Parfois l'indication concernant la divinité honorée est manquante et ne peut être déduite, comme pour le règlement de Tégée du IVe siècle av. J.-C. (LSS 31/ CGRN 65) ; c'est aussi le cas de LSCG 124/ CGRN 181, LSS 119/ CGRN 144 et LSCG 139. Quant au règlement du I $\mathrm{I}^{\mathrm{er}}$ siècle ap. J.-C. de Rhodes (LSS 108), nous ne savons pas s'il concerne un sanctuaire en I'honneur de Sarapis ou d'Asclépios.

[29] LSS 115/ CGRN 99.

[30] LSCG 95/ CGRN 203.

[31] LSCG 171/ CGRN 162.

[32] LSAM 12/ CGRN 212.

[33] LSS 59.

[34] LSAM 51/ CGRN 214.

[35] DECOURT \& TZIAPHALIAS 2015.

[36] LSS 91.

[37] LSAM 14.
[38] LSCG 151/ CGRN 86 ; LSCG 156/ CGRN 85.

[39] LSAM 29 / CGRN 71 et LSAM 18/ CGRN 211.

[40] SEG XXVIII 421/ LUPU 2005 n० 7/ CGRN 155.

[41] LSS 54/ CGRN 217.

[42] LSCG 55 ; DECOURT \& TZIAPHALIAS 2015.

[43] LSS 59, Délos, 116/115, sanctuaire de Zeus Kynthios et Athéna Kynthia ; LSS 108, Rhodes, I ${ }^{\text {er }}$ siècle ap. J.-C., sanctuaire de Sarapis ou d'Asclépios ; LSCG 139, Lindos, II ${ }^{\mathrm{e}}$ siècle ap. J.-C., sanctuaire en I'honneur de divinités orientales indéfinies; $L S C G 55$, Athènes, $\mathrm{II}^{\mathrm{e}}$ siècle ap. J.-C., sanctuaire de Mên ; LSS 91, Lindos, III ${ }^{\mathrm{e}}$ siècle ap. J.-C., sanctuaire d'Athéna.

[44] Antiphon, Sur le meurtre d'Hérode, V, 82 : « Vous savez tous, je pense, que bien des hommes dont les

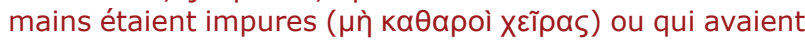
contracté quelque autre souillure (ä $\lambda \lambda$ o тı $\mu$ ía $\sigma \mu \alpha$ ), embarqués sur un vaisseau, ont entraîné dans leur perte ceux qui étaient en état de pureté à l'égard des dieux ; parfois aussi, de tels hommes, sans causer la mort d'autrui, lui ont fait courir les plus grands dangers ; souvent encore, lorsqu'ils se tenaient près des victimes, leur impureté (oủX ǒбıo ővTعs) est apparue en ce qu'ils empêchaient les sacrifices traditionnels de s'accomplir » (traduction Louis Gernet, CUF, 1923).

[45] Sur le vocabulaire varié de la souillure, se reporter à PARKER 1983, p. 1-17.

[46]Voir notice «Souillure » [par Marie Augier] dans BODIOU \& MEHL 2019. 
quarante-quatre jours) ou un lavage - une lustration -, ou un autre processus de purification. L'emploi d'un vocabulaire formulaire favorise donc la concision et celle-ci est redoublée par une construction elle-même elliptique qui conduit à un effacement de l'énonciateur. Les listes sont en effet introduites le plus souvent par une forme verbale à l'infinitif [47]. Suivent ensuite les prescriptions indiquées sous forme de listes. Dans la majorité des inscriptions du corpus [48], on emploie

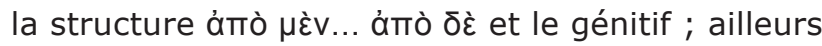
on emploie [49] la particule $\mu$ ǹ suivi de $\mu \eta \delta \bar{\varepsilon}$, répété autant de fois qu'il le faut.

Le large ambitus chronologique, le cadre géographique étendu et les sanctuaires variés concernés par les prescriptions cathartiques ne nuisent pas, à mon sens, à la cohérence d'un tel corpus, puisque des points communs saillants sont perceptibles entre les règlements : une structure formulaire, la fréquence de certaines causes de souillures et pour certaines d'entre elles une désignation formulaire. Il existe en outre un autre élément commun à toutes ces inscriptions : l'impureté et les interdits rituels ne semblent effectifs que dans l'espace sacré.

\section{PURETÉ RITUELLE ET ESPACE SACRÉ}

La lecture des inscriptions montre que la souillure n'a d'effet que dans l'espace sacré et pas ailleurs : une personne qui aura été en contact avec un mort, avec une accouchée ou qui aura eu des relations sexuelles ne sera donc pas souillée en soi, mais ne pourra simplement pas pénétrer tout de suite dans certains espaces sacrés [50].

La souillure est attachée à un lieu, à un territoire et n'est pas effective partout. La typologie des impuretés corporelles en est un premier indice. Si l'impureté corporelle découle par exemple du contact avec un mort ou avec une accouchée, de l'accouchement ou encore des relations sexuelles, il s'agit en fait d'évènements de la vie de tous les jours. Ces situations, qui n'ont rien d'exceptionnel, ne constituent une source d'impureté que lorsqu'une personne veut pénétrer dans I'espace sacré. Il leur faut alors se purifier, soit par le temps, soit avec des actes de purification précisés dans le règlement pour pouvoir être en état de pureté rituelle. Le fait que l'on peut facilement effacer une telle impureté indique que celle-ci n'affecte pas l'individu dans sa nature profonde.

La formulation des prescriptions cathartiques est un autre indice du caractère non pérenne et non essentiel de l'impureté notamment corporelle. C'est généralement la préposition apo qui est utilisée pour indiquer ce dont on doit se purifier sans autre précision. Cette formulation elliptique qui ne nomme pas les destinataires des prescriptions avait l'avantage de toucher le plus de personnes possibles et d'éviter ainsi qu'une personne impure ne pénètre dans le sanctuaire.

Le règlement du $\mathrm{II}^{\mathrm{e}}$ siècle av. J.-C. [51] qui était placé devant le sanctuaire d'une divinité syrienne à Délos illustre parfaitement ces quelques remarques :

áyaӨñ túxn· áyveúovtas

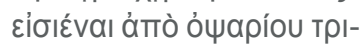

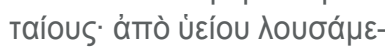

vov · ámò үuvaıkòs трıтаíoụ< $\varsigma>$

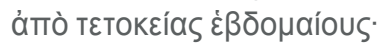

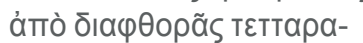

кобтаíous ámò үuvaıкहí-

wV ह่vataíous.

« À la Bonne Fortune! Entrer en étant pur : s'étant tenu éloigné des plats de poisson pendant trois jours, s'étant lavé après de la viande de porc, s'étant tenu éloigné d'une femme pendant trois jours, $d^{\prime}$ (une femme) ayant accouché pendant une semaine, d'(une femme) ayant avorté/ ayant eu une fausse couche pendant quarante jours, des menstruations pendant neuf jours. »

Les impuretés évoquées ici sont temporaires, puisqu'on ne demande qu'un délai plus ou moins long avant de recouvrer sa pureté rituelle. Celles-ci s'effacent parfois avec le temps, parfois avec une ablution, comme pour la consommation de porc. L'inscription, aussi elliptique soit-elle, indique en outre
[47] C'est le cas dans le règlement de Mégalopolis en Arcadie daté de 200 ap. J.-C. [LUPU 2005 n 7/ CGRN 155], qui était placé devant le sanctuaire d'Isis, Anoubis et

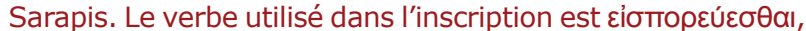
suivi du participe кaӨapißovтa; dans le règlement de Délos daté du $\mathrm{II}^{\mathrm{e}}$ siècle ap. J.-C. placé devant le sanctuaire des dieux Syriens [LSS 54], c'est le verbe દioléval, « entrer» qui est employé, précédé cette fois du participe áyveúovTas. L'idée est toujours la même : la pureté rituelle est nécessaire pour pouvoir pénétrer à l'intérieur du sanctuaire.

[48] LUPU 2005 n० 7/ CGRN 155 ; LSCG 171/ CGRN 162 ; LSCG 95/ CGRN 203 ; LSAM 12/ CGRN 212,
LSAM 14, LSAM 18/ CGRN 211, LSAM 29 / CGRN 71, LSAM 51/ CGRN 214, LSAM 84 ; LSS 119/ CGRN 144 ; LSS 54/ CGRN 217, LSS 59, 91 et 108 ; LSCG 55 ; LSS 4/ CGRN 217 ; DELCOURT \& TZIAPHALLIAS 2015 ; LSCG 124/ CGRN 181 ; LSCG 94/ CGRN 173.

[49] LSCG 68/ CGRN 126 ; LSAM 14, LSAM 29/ CGRN 71 ; LSAM 6 ; LSAM 84 ; LSS 91 et 106 ; LSCG 154/ CGRN 148 ; LSCG 156/ CGRN 85 ; LSCG 94/ CGRN 173 ; LSS 59 ; LSCG 55.

[50] Sur cette question, AUGIER 2015 et 2016.

[51] LSS 54/ CGRN 217. 
plus clairement cette séparation entre deux espaces : I'espace sacré et l'espace qui ne l'est pas, marqué par le verbe عíøı́vaı [52].

Parfois la séparation entre les deux espaces est évoquée plus précisément encore, par la mention d'un lieu réservé aux pratiques purificatoires, comme dans le règlement du sanctuaire d'Athéna Nikèphoros à Pergame, daté d'après 133 av. J.-C. [53]. Au sujet des funérailles, on peut lire ce qui suit :

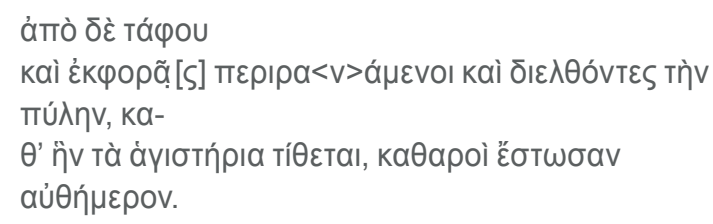
et au convoi funèbre, après s'être aspergés et avoir passé la porte auprès de laquelle se tiennent les instruments de lustration, soient purs le jour même [54]. »

Le terme générique àjıotńpı reste malheureusement assez vague et les données archéologiques ne permettent pas de préciser les choses, mais il indique que les fidèles pouvaient procéder à certaines ablutions à l'entrée de l'espace sacré. Aussi, outre la porte qui marque un lieu de passage qui permet d'entrer dans l'espace sacré délimité par le péribole, les instruments de lustration permettent d'entrer en état de pureté rituelle après avoir pénétré un autre espace : I'espace sacré nécessitant une pureté rituelle distinct de I'espace du dehors où cette impureté n'empêche pas d'agir. Certains documents mentionnent des perirrhanteria. Pour entrer dans le sanctuaire d'Athéna à Lindos d'après une inscription du III $^{\mathrm{e}}$ siècle ap. J.-C. [55], la pureté rituelle est exigée après avoir passé les portes et les perirrhanteria :

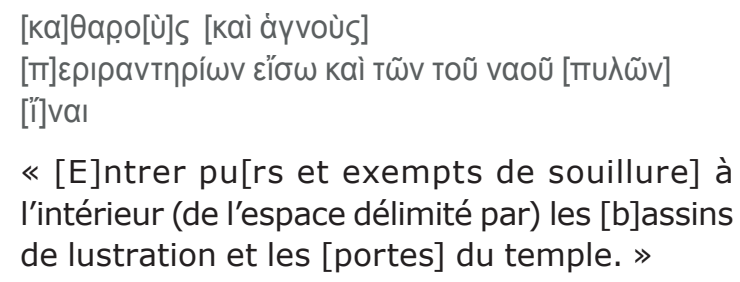

De la même manière dans le règlement des mystères d'Andanie daté de 92 av. J.-C. [56], on peut lire :

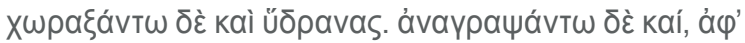

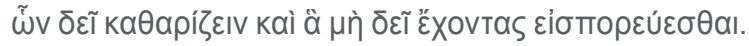

«Que les hiéroi [oi ícpoì mentionnés plus haut] mettent en place aussi des vases à lustration. Et qu'il soit inscrit aussi de quoi il faut se purifier et quels objets il ne faut pas avoir pour entrer. »[57]

Le règlement prévoit d'afficher des prescriptions qui indiqueront les souillures nécessitant une purification, visiblement au moyen des hydranai. Ces vases à lustration, outre leur utilité, symbolisent également la limite après laquelle l'impureté rituelle est effective.

Des bassins de lustration ont été trouvés dans de nombreux sanctuaires [58], mais la question de leur usage réel reste problématique. À quoi ces instruments de lustration ou ces bassins servaient-ils vraiment? S'agissait-il des ablutions demandées dans les prescriptions cathartiques ou d'ablutions pratiquées quel que soit l'état de pureté rituelle de l'individu qui souhaitait pénétrer dans le sanctuaire ? Il est difficile de répondre à cette question, mais on peut se demander si certaines purifications ne devaient pas être effectuées chez soi, notamment lorsqu'on demandait de s'« être lavé de la tête aux pieds » [59].

La variété des techniques purificatoires [60] va en effet plutôt dans le sens d'une purification préalable, à laquelle peut s'ajouter une purification habituelle au moyen d'ablutions pratiquées à l'entrée du sanctuaire. Le procédé le plus courant pour recouvrer sa pureté rituelle est de laisser partir la souillure avec le temps. Mais parfois cette attente est remplacée ou complétée par d'autres techniques. Généralement, on demande de se laver : il suffit le plus souvent, particulièrement après les relations sexuelles [61]. C'est le verbe louomai qu'on emploie dans ce cas, mais sans indiquer ce qu'il fallait laver : les mains seules, d'autres parties du corps ? Le corps en entier ? La précision « des pieds à la tête » donnée dans certains règlements ne
[52] Sur cette frontière entre deux espaces et la nécessité d'adapter son comportement et sa tenue en pénétrant dans le temenos, GRAND-CLÉMENT 2019.

[53] LSAM 12 / CGRN 212.

[54] Cette précision semble indiquer que l'impureté, qui se serait effacée certainement avec le temps, a disparu plus rapidement grâce à l'ablution rituelle.

[55] LSS 91.

[56] LSCG 65/ CGRN 222.

[57] Traduction DESHOURS 2006 légèrement modifiée. [58] Sur les perirrhanteria, DuCAT 1964 et PIMPL 1997.
[59] LUPU $2005 \mathrm{n}^{\circ}$ 7/ CGRN 155 = SEG XXVIII, 421 (vers 200 av. J.-C.), règlement de pureté pour l'entrée dans le sanctuaire d'Isis, Anoubis et Sarapis à Mégalopolis : on y demande de s'être lavé de la tête aux pieds après la consommation de certains aliments ; dans le règlement $L S C G 55$ ( $\mathrm{II}^{\mathrm{e}}$ siècle ap. J.-C., culte de Mên à Sounion), c'est après des relations sexuelles et les menstrues.

[60] Sur les techniques de purification, Augier 2017a. [61] Par exemple : SEG XXVII, 421, LSS 115/ CGRN 99, LSCG 124/ CGRN 181, LSAM 12/ CGRN 212 et LSAM 18/ CGRN 211, LSS 91. 
peut être généralisée à tous les sanctuaires ; aussi est-il difficile de savoir si les instruments de lustration se trouvant dans le sanctuaire suffisent. Parfois, les règlements disent simplement qu'on peut entrer après s'être « purifié », sans préciser ce qui est entendu par ce terme très général [62].

Dans une inscription de Méonie en Lydie, datée de 147/146 av. J.-C., on peut lire que l'hetaira [63] ne peut pénétrer dans le sanctuaire de la Déesse-Mère que « le troisième jour [après une relation sexuelle], après s'être entièrement purifiée comme il est habituel » [64] :

\section{غ̇таípa трІт-}

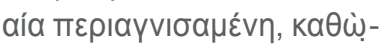

ऽ عї̈ıбтаı.

Rien ne permet de savoir à quelle pratique le règlement fait allusion.

Des procédés spécifiques étaient parfois indiqués dans la réglementation. L'eau de mer pouvait être utilisée, comme pour purifier la maison du mort après ses funérailles à Iulis [65]. Dans un règlement de Cos du $\mathrm{II}^{\mathrm{e}}$ siècle ap. J.-C. [66], il fallait se laver et s'oindre

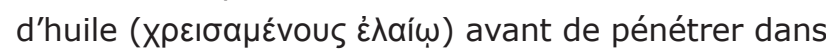
le sanctuaire après avoir eu des relations sexuelles dans le cadre conjugal.

Parfois les techniques de purifications pouvaient être beaucoup plus complexes. À Cos, d'après deux règlements du III siècle av. J.-C. [67], deux prêtresses de Déméter et le prêtre de Zeus Polieus n'avaient pas le droit, pendant leur charge, d'entrer dans la maison où quelqu'un était mort et où une femme avait accouché ou avorté, ou encore ne pouvaient pas toucher de bête morte [68]. Ces règles s'expliquent par leur rôle prépondérant dans l'accomplissement des rituels. Si ces agents du culte faisaient malgré tout une des choses interdites, le procédé de purification qu'ils devaient suivre était alors très complexe ; voici ce qui était exigé du prêtre de Zeus Polieus [69] :

[62] Par exemple : LSAM 18/ CGRN 211, LSS 91 et LSS 59.

[63] Dans le CGRN $\left(n^{\circ} 211\right)$, la traduction « prostituée » est proposée pour le terme غ̇тaípa, mais il me semble ici que la précision du type de partenaire porte peut-être sur la distinction entre les relations sexuelles dans le cadre du mariage légitime et celles qui n'ont pas lieu dans ce cadre sans que l'on puisse conclure que dans ce second cas il s'agisse de prostituées. Un autre règlement fait précisément cette distinction ; dans une inscription de Métropolis du IV siècle av. J.-C. (LSAM 29/ CGRN 71), on peut lire : « On est pur [...] en s'étant tenu éloigné de relations avec sa propre femme pendant 2 jours, avec une hétaïre pendant 3 jours. »

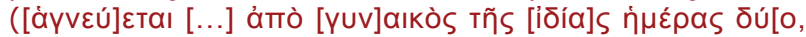

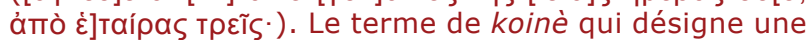

aỉ ठદ́ Tí ka тoúт-

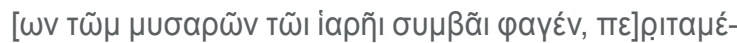

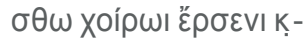

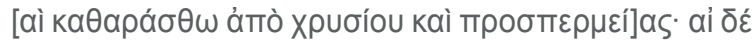
Tí $k \alpha$ Tथ̃v å $\lambda<\lambda>\omega v$ T!-

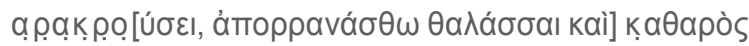
हैंTT. [70]

« S'il arrive [au prêtre de manger une de ces choses impures, qu'il] coupe les extrémités d'un porcelet mâle e[t qu'il se purifie avec une coupe d'or et une prosperm]ia [71] ; si quelque chose d'autre le pousse hors du droit [chemin, qu'on répande de l'eau de mer (sur lui) et] qu'il soit pur. »

Le sang du porcelet servait à purifier le prêtre, car selon le règlement des prêtresses de Déméter, il devait être répandu tout autour du prêtre. La coupe d'or devait contenir les graines pour l'aspersion. Peu de règlements sont aussi précis que celui-ci, mais il a pour particularité de concerner des agents du culte. La question du lieu où les purifications étaient pratiquées se pose également : puisqu'il fallait utiliser le sang d'un porcelet, le rituel de purification devait-il avoir lieu dans le sanctuaire ou fallait-il I'accomplir avant d'y retourner ? La question est difficile, mais il est intéressant que ces documents rappellent l'importance de la distinction entre deux espaces : un espace où les impuretés évoquées dans les prescriptions ne grevaient pas les actions des individus et un autre où ce qui n'est pas forcément exceptionnel devient une cause d'impureté.

Attachons-nous à présent aux impuretés corporelles en nous demandant quelles indications elles donnent, en creux, sur des actes interdits dans I'espace sacré.

\section{TYPOLOGIE NON EXHAUSTIVE DES IMPURETÉS CORPORELLES}

L'impureté corporelle est évoquée dans la majorité des règlements. Elle est plus ou moins grave en fonction

prostituée est employé quant à lui dans l'inscription LSS 91.

[64] LSAM 18/ CGRN 211, I. 13-15.

[65] LSCG 97/ CGRN 35.

[66] LSCG 139.

[67] LSCG 154/ CGRN 148 et LSCG 156/ CGRN 85.

[68] Sur la pureté des prêtres et des prêtresses, notamment à Cos, WILGAUX 2009, p. 231-242.

[69] LSCG 156/ CGRN 85, I. 13-16.

[70] La restitution est possible par comparaison avec un autre règlement mentionnant les purifications auxquelles devaient se soumettre les prêtresses de Déméter Olympia et Isthmos à Cos : LSCG 154/ CGRN 148.

[71] Aspersion de graines. 
de sa typologie, mais aussi en fonction des sanctuaires, certains se démarquant par leur sévérité. En indiquant de quoi le corps doit avoir été éloigné pendant une durée spécifique, les prescriptions cathartiques indiquent aussi ce qui ne peut avoir lieu dans le sanctuaire. Il ne s'agit pas ici d'entrer dans le détail de ces impuretés corporelles [72], mais de les lister rapidement pour savoir ce qu'on ne faisait pas dans l'espace sacré.

Le cadavre [73], par les miasmes qu'il dégage, peut contaminer toutes sortes d'espaces. Aussi, le contact avec un mort [74] est-il une des causes les plus courantes d'impureté corporelle [75]. Il nécessite un délai pour recouvrer sa pureté avant de pénétrer à nouveau dans un sanctuaire, mais l'impureté corporelle du cadavre touche aussi sa maison qui doit être purifiée [76]. Les prescriptions distinguent plusieurs types de contact avec le mort. Avoir pris part aux funérailles d'un proche est ce qui souille le plus, car cela implique généralement les personnes vivant sous le même toit [77]. Les proches sont donc exposés directement aux miasmes du mort et à la contamination de la maison pendant l'exposition du cadavre, mais ils prennent aussi part à la procession vers le monument funéraire. Parmi les proches, les femmes qui donnent les soins au cadavre sont évidemment pleinement concernées. En revanche, les personnes qui ne font que s'approcher du monument funéraire, et qui auront simplement assisté au convoi funèbre seront moins souillées.

Cette distinction est ainsi précisée dans le règlement du sanctuaire d'Athéna à Lindos [78] :

\section{[ảm] kñóous oíkíou $\mu \alpha^{\prime}$.

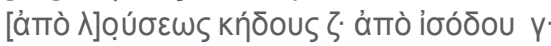

« S'être tenu éloigné des funérailles d'un proche depuis quarante-et-un jours, du bain d'un cadavre depuis sept jours [79], du monument funéraire depuis trois jours. »

[72] Pour une typologie des impuretés corporelles, WÄCHTER 1910, MOULINIER 1950, PARKER 1996, AUgier 2015.

[73] Voir notice « Cadavre » [par Reine-Marie Bérard] dans BOdIOU \& MEHL 2019.

[74] Sur les règlements funéraires: FRISONE 2000 ; sur la souillure et la mort : ECK 2012.

[75] LSS 31/ CGRN 65 ; LSS 115/ CGRN 99 ; LSAM 29/ CGRN 71 ; LSCG 124/ CGRN 181 ; LSAM 18/ CGRN 211 ; LSAM 12/ CGRN 212 ; LSAM 51/ CGRN 214 ; LSCG 139 ; LSCG 55 ; LSS 91 ; LSAM 14.

[76] LSCG 97/ CGRN 35.

[77] Dans les inscriptions, on ne précise pas le sexe des personnes concernées par cette impureté, mais on
Les délais pour recouvrer sa pureté, même si ce règlement est particulièrement sévère, sont tout à fait révélateurs de l'échelle de gravité en fonction de la proximité avec le cadavre. Le risque d'introduire dans le sanctuaire l'impureté associée au cadavre explique toutes les précautions prises lorsqu'un animal est sacrifié, mais aussi certains interdits, comme celui de mourir et d'être enterré sur l'île de Délos, consacrée à Apollon, qui a conduit au ve siècle av. J.-C. à une purification générale de l'île. Les morts ont été déterrés et enterrés sur l'île voisine de Rhénée [80].

Si on ne peut mourir sur lî̂le de Délos, on ne peut pas non plus y naître, puisque les femmes enceintes comme les morts déjà enterrés ont dû quitter lî̂le lors de sa purification. La femme en couches souille en effet l'endroit où elle donne la vie [81]. La maison avec son toit et ses murs est un espace clos qui conserve la souillure. Un règlement de Cyrène datant du Ive siècle av. J.-C. [82] est assez clair sur ce sujet :

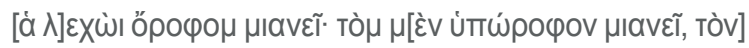

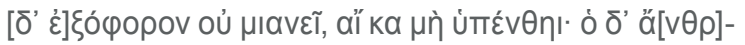

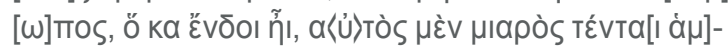

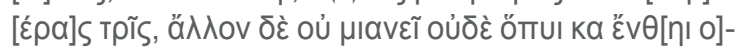

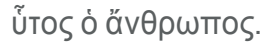

« La femme en couches souillera le toit : [elle souillera qui est sous le toit, mais] ne souillera pas qui est en-dehors, s'il n'y pénètre pas. Et la personne qui est à l'intérieur sera personnellement soumise à la souillure pendant trois jours, mais ne la transmettra pas à autrui, où qu'elle se rende. » [83]

Les délais pour recouvrer sa pureté après une naissance divergent justement en fonction de la personne touchée par la souillure : I'accouchée elle-même, qui subissait directement l'accouchement, avait besoin d'un temps plus long pour recouvrer sa pureté rituelle, tandis que ceux qui étaient en contact avec l'accouchée et qui subissaient de manière indirecte la souillure

indique simplement que le fait de prendre part à telle ou telle partie des funérailles entraîne une impureté rituelle.

[78] LSS 91, III ${ }^{\mathrm{e}}$ ap. J.-C.

[79] La durée de 7 jours pour le bain du cadavre paraît étrangement courte dans ce règlement.

[80] Thucydide, III, 104.

[81] Règlements évoquant la naissance et l'avortement ou la fausse couche comme impureté corporelle : LSS 115/ CGRN 99 ; SEG XXVIII 421/ LUPU $2005 \mathrm{n}^{\circ} 7 /$ CGRN 155 ; LSCG 124/ CGRN 181 ; LSCG 171/ CGRN 162 ; LSS 54/ CGRN 217 ; LSAM 20/ CGRN 191 ; LSS 119/ CGRN 144 ; LSAM 51/ CGRN 214 ; LSCG 139 ; LSCG 55; LSS 91 ; LSAM 14/ SEG 4, 681. Sur l'accouchement : MEHL 2009 ; BODIOU \& BRULÉ 2011.

[82] LSS 115/ CGRN 99.

[83] Traduction DOBIAS LALOU 1988. 
devaient patienter moins longtemps. Ces dernières personnes ne pouvaient quant à elles en souiller d'autres.

La pratique de l'avortement [84] est connue en Grèce ancienne, mais on la distingue mal dans les prescriptions cathartiques de la fausse couche. Il semblerait que du point de vue de l'impureté rituelle ces deux évènements soient confondus. Dans le cas d'un avortement ou d'une fausse couche, la durée pour recouvrer sa pureté est plus longue que pour une naissance, car il faut intégrer la mort du fœetus et il s'agit généralement d'une durée d'une quarantaine de jours quel que soit le sanctuaire. Deux règlements évoquent également la pratique de l'exposition qui souille autant que l'avortement ou la fausse couche [85], la finalité de cette pratique étant la mort du nouveau-né.

Enfin, les relations sexuelles [86] font partie des sources d'impureté corporelle les moins graves et les plus fréquentes [87]. Malgré tout, le vocabulaire des prescriptions « sexuelles » est on ne peut plus elliptique : soit un terme générique comme ouvouoía ou tà áppodíoı est employé, soit on désigne le partenaire sexuel : ámò yuvaı́ós par exemple, que l'on comprend « après une relation sexuelle avec une femme » [88].

En outre, ce type d'impureté est la plus facile à effacer, puisque le plus souvent un simple lavage suffit après une relation sexuelle ou un délai très court. Parfois, des distinctions de durée sont faites en fonction du partenaire sexuel : si la relation a lieu dans le cadre du mariage, la durée est ainsi moins longue que s'il s'agit d'une relation hors de ce cadre, avec une prostituée par exemple. Le règlement de Pergame évoqué plus haut [89] est en ce sens tout à fait remarquable, car il distingue les deux cas de figures aussi bien pour l'époux que pour l'épouse :

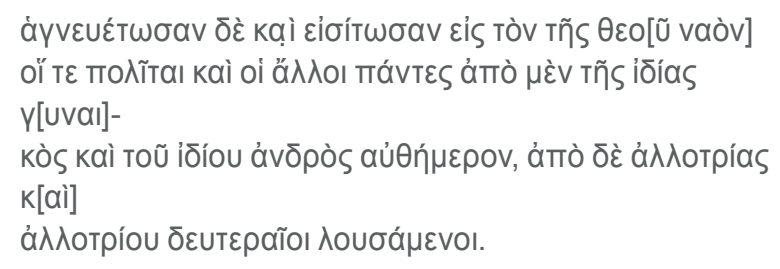

[84] Voir notice « Avortement » [par Lydie Bodiou] dans BODIOU \& MEHL 2019.

[85] LSS 119/ CGRN 144 et LSAM 84.

[86] Sur la sexualité antique, voir BOEHRINGER 2018.

[87] LSS 31/ CGRN 65 ; SEG XXVIII 421/LUPU 2005, $\mathrm{n}^{\circ} 7 /$ CGRN 155 ; LSCG 55; LSAM 29/ CGRN 71 ; LSAM 18/ CGRN 211 ; LSAM 12/ CGRN 212 ; LSAM 20/ CGRN 191 ; LSAM 51/ CGRN 214 ; LSAM 14 ; LSCG 151/ CGRN 86 ; LSCG 156/ CGRN 85 ; LSCG 124/ CGRN 181 ; LSCG 171/ CGRN 162 ; LSS 54/ CGRN 217 ; LSCG 95/ CGRN 203 ; LSS 59 ; LSS 108 ; LSCG 139 ; LSS 91 ; LSS 119/ CGRN 144 ; LSS 115/ CGRN 99. Voir EIDINOV 2011.
« Les citoyens et tous les autres doivent être purs et entrer dans le temple de la déesse en n'ayant pas eu de relation avec leur propre épouse et leur propre époux le jour même, et deux jours après s'être lavés en n'ayant pas eu de relation avec une autre et un autre. »

Il est tout à fait notable que le règlement prévoit les relations extra-conjugales pour les femmes citoyennes également, lorsqu'on sait qu'à Athènes les gunaikes adultères ne pouvaient avoir accès au sanctuaire pendant dix ans [90]. Cela ne signifie pas que l'adultère n'était pas condamné à Pergame, mais simplement que le règlement se veut le plus détaillé possible par rapport aux souillures sexuelles.

Il est, en outre, interdit d'avoir un rapport sexuel dans un sanctuaire. Hérodote, dans le livre II de son Enquête, fait des Égyptiens et des Grecs les seuls peuples à refuser les relations sexuelles dans les sanctuaires, tout en donnant la primauté de l'interdit à l'Égypte : «Ce sont également les Égyptiens qui les premiers se sont fait une loi de ne pas s'unir à des femmes dans des sanc-

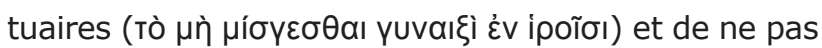
y entrer en quittant des femmes avant de s'être lavés

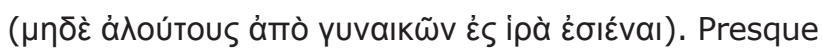
tous les autres hommes, Égyptiens et Grecs mis à part, s'unissent aux femmes dans les lieux saints ou, en se levant de leur lit, entrent dans un sanctuaire sans s'être lavés au préalable. » [91].

À partir du $\mathrm{II}^{\mathrm{e}}$ siècle av. J.-C., une autre impureté corporelle apparaît dans des règlements assez marginaux par l'origine étrangère de leur culte [92] : I'impureté liée aux menstruations. Les règles entraînaient une contamination assez longue, puisque les femmes devaient attendre entre sept et neuf jours après les menstrues pour pénétrer à nouveau dans le sanctuaire, ce qui leur interdisait l'entrée pendant près de la moitié du mois. Nous pouvons considérer que cet interdit est marginal dans le monde grec, car les sources sont en général assez silencieuses sur les règles, les Grecs ne semblant pas connaître de tabou lié à ce phénomène.

[88] Il est intéressant de noter qu'on ne désigne pas qui est touché par cette impureté rituelle, ni même le sexe de cette personne.

[89] LSAM 12/ CGRN 212.

[90] Démosthène, Contre Nééra, 85-87 ; Eschine, Contre Timarque, 183.

[91] Hérodote, II, 64 (traduction P.-E. Legrand, CUF). Pausanias quant à lui évoque plusieurs fois cet interdit comme lorsqu'il raconte dans sa Description de la Grèce de quelle manière Artémis se venge de la prêtresse Comaithô utilisant son sanctuaire comme chambre nuptiale avec Mélanippe en décimant les habitants du voisinage (VII, 19, 1-6).

[92] Sur cette question, Augier 2017b. 
L'allaitement se trouve quant à lui dans deux des règlements évoquant la menstruation [93]. Mais le caractère encore plus marginal de cet interdit ne permet pas de savoir si c'est le lait maternel ou le bruit occasionné par la présence d'un enfant en bas âge qui en est à I'origine. Il n'y a en effet pas non plus de tabou associé en Grèce au lait maternel.

Sans être entré dans le détail de ces quelques impuretés corporelles, on peut néanmoins constater que les actions rejetées à l'extérieur du sanctuaire touchent à la fois à la vie au sein de l'oikos, mais aussi au sein de la communauté civique en général : naissance, sexualité, mort. Toutes les « activités » liées au corps dans les interactions sociales n'étaient pas compatibles avec la pratique des rituels, comme s'il fallait conceptualiser par ces interdits la séparation entre deux espaces. Néanmoins à aucun moment on ne peut voir, dans le dispositif mis en place dans les prescriptions cathartiques, de jugement sur ces pratiques interdites dans les sanctuaires : il y a simplement une différenciation du rapport au corps dans l'espace sacré, par rapport à l'espace qui ne l'est pas. Le corps n'est pas en ce sens impur en essence, mais parce qu'il a été en contact avec des vecteurs d'impureté.

\section{LES INTERDITS ALIMENTAIRES}

Les prescriptions cathartiques indiquent aussi parfois des interdits alimentaires. La consommation de certains aliments devait être évitée avant de pénétrer dans certains sanctuaires et si elle avait eu lieu, un délai ou une technique de purification similaire à ceux qui suivent une impureté corporelle devaient se dérouler. L'interdit de manger de la viande en général [94], ou certaines viandes comme le porc [95], la chèvre [96] ou le cœur [97] se trouve dans certains documents. Et pourtant, il n'y avait pas d'animaux impurs en Grèce ancienne [98], et la consommation de viande faisait partie intégrante des rituels pratiqués dans le sacrifice. Aussi pourrions-nous expliquer ces interdits, qui se trouvent par ailleurs dans des règlements assez récents, par une influence pythagoricienne [99]. Le végétarisme est en effet traditionnellement attaché aux personnages de Pythagore ou d'Empédocle, tout comme un autre interdit alimentaire qui se trouve également dans certains de ces règlements : le rejet de la fève. La consommation de viande est comparable au meurtre, car les animaux comme les hommes possèdent une âme selon la doctrine pythagoricienne de la métempsychose ; les hommes peuvent se réincarner en animal ou en homme [100]. Les Anciens donnaient au rejet des fèves plusieurs explications, la plus courante étant que les fèves contiennent les esprits des morts [101] ; manger des fèves reviendrait donc à manger la tête de ses parents [102] Une fève découpée a d'ailleurs l'odeur de la semence selon Porphyre [103] : Iorsque I'on plante une fève et qu'on regarde la racine une fois qu'elle a poussé, celle-ci ressemble soit à une tête d'enfant, soit à des parties génitales.

Le poisson [104] est également rejeté dans des règlements placés devant des sanctuaires qui ont été attribués, en raison de cette mention, à la déesse Atargatis, même si les Pythagoriciens s'en abstiennent aussi selon Plutarque [105]. Le poisson était en effet
[93] LSS 91 et SEG 8, 639 / LSS 119/ CGRN 144.

[94] LSS 59, Délos, 116/115 av. J.-C. (Zeus Kynthios et Athéna Kynthia).

[95] LSS 54/ CGRN 217, Délos, II ${ }^{\mathrm{e}}$ siècle av. J.-C. (Agartatis) ; LSCG 55, Sounion, $\mathrm{II}^{\mathrm{e}}$ siècle ap. J.-C. (Mên) ; LSS 55, Délos, $\mathrm{II}^{\mathrm{e}}$ siècle ap. J.-C. (divinités orientales). Voir aussi LSS 63/ CGRN 27, Thasos, 440 av. J.-C. (Héraclès) et LSS 73/ CGRN 178, Thasos, $\mathrm{II}^{\mathrm{e}}$ siècle av. J.-C. (Peithô).

[96] SEG XXVIII 421/ LUPU $2005 \mathrm{n}$ 7/ CGRN 155, Mégalopolis, 200 av. J.-C. (Isis, Sarapis et Anoubis) LSCG 139, Lindos, II ${ }^{\mathrm{e}}$ siècle ap. J.-C. (Culte oriental) ; LSS 55, Délos, II ${ }^{\mathrm{e}}$ siècle av. J.-C. (divinités orientales). Voir aussi LSS 57/ CGRN 174, Délos, II ${ }^{\mathrm{e}}$ siècle av. J.-C. (divinités sémitiques) ; LSS 58/ CGRN 216, Délos, 100 av. J.-C., (Poséïdon) ; LSS 63/ CGRN 27, Thasos, 440 av. J.-C. (Héraclès) ; LSS 73/ CGRN 178, Thasos, II ${ }^{\mathrm{e}}$ siècle av. J.-C. (Peithô) ; LSS 74/ CGRN 23, Thasos (Héra) ; LSS 91, Lindos, III $^{\mathrm{e}}$ siècle ap. J.-C.

[97] LSS 108, Rhodes, I $\mathrm{I}^{\mathrm{er}}$ siècle ap. J.-C. (Sarapis ou Asclépios) ; I'interdit du cœur pour certaines victimes se trouve aussi dans un règlement d'Éphèse du III ${ }^{e}$ siècle ap. J.-C. (LSS 121) qui précise quelles victimes devaient fournir les prytanes en distinguant celles qui sont sans

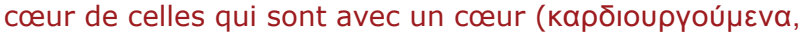

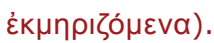

[98] Sur cette question, PARKER 1983 ou 1996, p. 357 et suiv.

[99] Sur les interdits et tabous alimentaires des Pythagoriciens, DETIENNE \& VERNANT 1979, p. 76-114, Osborne 1995, p. 214-224, GaRnsey 1999, BeTEGH 2014, p. $149-166$.

[100] D'après Plutarque (Moralia, 353), les prêtres égyptiens évitaient également le porc, qu'ils considéraient comme sale.

[101] Pline, Histoire Naturelle, 18, 119 ; Diogène Laërte $8,24$.

[102] Athénée, 65f.

[103] Porphyre, Vie de Pythagore, 44.

[104] LSS 54/ CGRN 217, Délos, II ${ }^{\mathrm{e}}$ siècle av. J.-C. (Agartatis) ; LSCG 95/ CGRN 203, Délos, après 166 av. J.-C. (Artémis).

[105] Plutarque, Moralia, 728-730 ; cf. aussi Diogène Laërte 8, 35. 
consacré à la déesse dans son culte, comme nous pouvons le lire dans une inscription de Smyrne, qui précise les sanctions promises à quiconque consommerait du poisson [106]. Enfin, l'ail est rejeté du sanctuaire de Mèn à Sounion [107]. Les interdictions de consommer ces aliments avant de pénétrer dans le sanctuaire indiquent de manière générale - sauf cas particulier comme dans le culte d'Atargatis -, en creux, ce qui ne pouvait être consommé à l'intérieur du sanctuaire.

\section{LES VÊTEMENTS ET OBJETS INTERDITS}

L'accès au sanctuaire était également limité par d'autres types d'interdits : les interdits vestimentaires ou touchant à certains objets. La couleur, la matière ou la valeur des vêtements pouvait ainsi être contrôlées, et on ne pouvait emporter sur soi ou avec soi des objets tels que des bijoux ou des armes [108]. Enfin, on interdisait parfois de faire entrer des animaux. Ces interdits n'ont pas tous la même explication ; certains s'expliquent par le rejet du cadavre hors du sanctuaire - sauf celui qui a fait l'objet d'un sacrifice -, tandis que les autres trouvent leur origine dans le souci

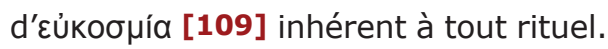

Les interdits concernant la matière de certains vêtements relèvent de la première catégorie. Dans plusieurs sanctuaires, les vêtements en cuir [110] ou les sandales sont prohibés. C'est notamment le cas du sanctuaire d'Alectronè à Ialysos, d'après une inscription [111] datée de 300 av. J.-C. : «Qu'on ne porte

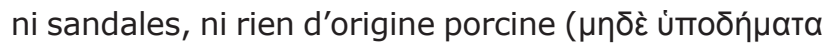

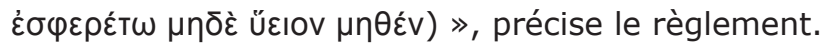
Et plus bas, les sanctions sont données :

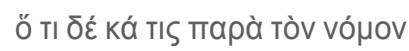

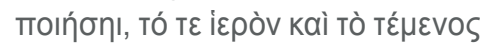

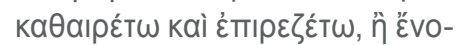

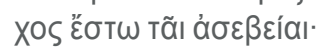

[106] LSAM 17, I $^{\text {er }}$ siècle av. J.-C.

[107] LSCG 55, Sounion, $\mathrm{II}^{\mathrm{e}}$ siècle ap. J.-C.

[108] Sur ce type d'interdits notamment dans les sanctuaires de Déméter et Despoina, GRAND-CLÉMENT 2019 ; sur les interdits vestimentaires, MILLS 1984.

[109] L'eukosmia désigne le «bon ordre » attendu lors des rituels. Certains magistrats peuvent être chargés de le faire respecter comme les pédonomes et les gynéconomes selon Aristote (Politique, 1299b) ; sur cette notion dans le règlement des Mystères d'Andanie (LSCG 65/ CGRN 222), DESHOURS 2006, p. 99-108.

[110] Lors des Mystères d'Andanie [LSCG 65/ CGRN 222], on ne peut porter de sandales en cuir provenant d'animaux offerts en sacrifice aux dieux.
«Si quelqu'un agit à l'encontre de la loi, qu'il purifie le sanctuaire et le temenos et qu'il y fasse un sacrifice ou il sera accusé d'impiété ».

Le cuir rappelle les interdits liés au cadavre, tandis que la précision sur le porc renvoie aux interdits alimentaires associés à cet animal dans certains sanctuaires. L'introduction de telles matières entraîne bien une impureté rituelle comme le précisent les sanctions requises en cas de non-respect des prescriptions. Le rejet du cuir de porc n'est pas la seule précision que I'on peut trouver dans la règlementation : dans certains cas il s'agit de la chèvre, comme dans le règlement récent de Lindos évoqué plus haut [112]. Voici les interdits vestimentaires dans ce règlement :

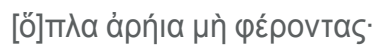

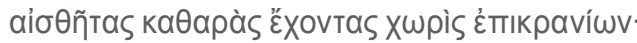

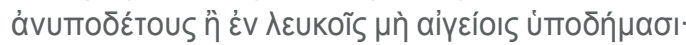

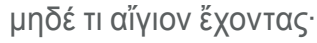

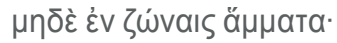

« Ne pas porter d'armes de guerre, mais avoir des vêtements purs, tête nue, en allant pieds nus ou bien dans des chaussures blanches, mais pas en chèvre - ne rien porter qui soit en chèvre, ni de nœud à la ceinture. »

Le rejet des armes et les indications de couleur seront explicités ensuite, mais on note déjà que ce qui ne convient pas dans le sanctuaire, plus que le cuir, est la chèvre en elle-même, qui est rejetée de plusieurs sanctuaires grecs [113].

La précision des pieds nus se trouve également dans plusieurs documents [114] qui ne précisent pas d'animal tabou dans le sanctuaire ; c'est ce même interdit du cuir qu'il faut peut-être comprendre dans ces cas, même si cela peut s'apparenter également à des interdits somptuaires ou à un contact direct avec la terre. Dans un règlement du sanctuaire des divinités égyptiennes de Délos du II ${ }^{\mathrm{e}}$ siècle av. J.-C. [115], c'est la laine qui est proscrite pour les hommes, seuls admis, qui entrent dans le sanctuaire.

\section{[111] LSCG 136/ CGRN 90.}

[112] Sanctuaire d'Athéna, III i siècle ap. J.-C. [LSS 91]. [113] SEG XXVIII 421/LUPU 2005, n 7 / CGRN 155 ; LSCG 139 ; LSS 91.

[114] Les pieds nus sont requis à Lykosoura dans le cadre des Mystères de Despoina (LSCG 68/ CGRN 126, $\mathrm{III}^{\mathrm{e}}$ siècle av. J.-C.) ; à Erésos dans un sanctuaire les sandales et les vêtements de peau sont interdits (LSCG 124/ CGRN 181, II siècle av J.-C.), ce qui relie bien le fait d'aller pieds nus et le rejet du cuir. Les autres règlements mentionnant les pieds nus sont : LSAM 6, LSS 59, LSAM 14, LSCG 65/ CGRN 222.

[115] LSS 56. 
L'eukosmia [116] est la seconde explication aux interdits portant sur des vêtements, des objets et même des animaux. Il s'agit de faire en sorte que les rituels se passent dans le calme et dans le respect de la divinité. C'est pourquoi des magistrats peuvent être désignés pour encadrer les participants et vérifier qu'ils respectent les interdits. Le règlement des Mystères d'Andanie [117] est en ce sens un bon exemple : non seulement des gynéconomes doivent veiller à ce que les femmes qui participent aux Mystères respectent les interdits vestimentaires et marchent dans le bon ordre lors de la procession, mais il mentionne toute une liste d'« encadrants » : hieroi et hierai, prêtre, secrétaire des synèdres, agoranome, les Dix, épimélètes, trésoriers, juges... Le rôle et la tenue, dans tous les sens du terme, de tous les participants sont précisés dans le règlement et des sanctions sont prévues en cas de comportements inappropriés.

Le calme peut être perturbé d'abord en introduisant par exemple des animaux dans le sanctuaire [118]. C'est pourquoi dans les prescriptions placées à l'entrée du sanctuaire d'Aléctronè à Ialysos [119], outre le cuir évoqué plus haut, on interdit aussi l'accès aux animaux qui pourraient paître dans le sanctuaire de la déesse :

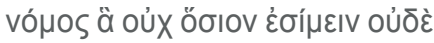

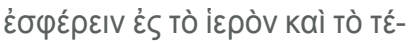

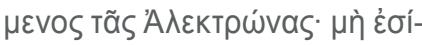

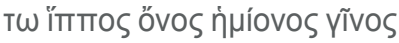

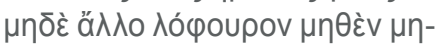

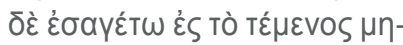

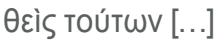

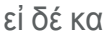

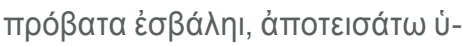

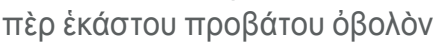

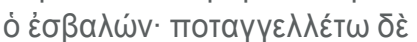

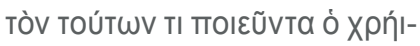

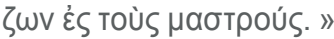
«Loi : Voici ce qu'il n'est pas permis de faire entrer ou d'apporter dans le sanctuaire et le domaine d'Alektryona : que n'entrent ni cheval,

[116] Sur le souci d'eukosmia dans le règlement des Mystères d'Andanie, Deshours 2006, p. 99-108.

[117] LSCG 65/ CGRN 222.

[118] LSCG 124/ CGRN 181 et LSCG 136/ CGRN 90.

[119] LSCG 136/ CGRN 90, 300 av. J.-C.

[120] Le règlement d'Erésos du $\mathrm{II}^{\mathrm{e}}$ siècle av. J.-C. [LSCG

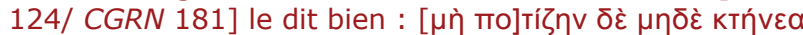

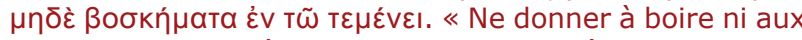
troupeaux ni au bétail dans l'espace sacré. »

[121] LSS 33/ CGRN 127 (I'aulos est interdit), LSCG 124/ CGRN 181 (armes de guerre et objets en fer ou en bronze), LSS 59, LSAM 77.

[122] LSCG 94/ CGRN 173.

[123] LSAM 77. ni âne, ni mule, ni bardot, ni aucune autre bête de somme, et qu'on n'introduise dans le domaine aucun de ces animaux [...] ; si quelqu'un pousse dedans du petit bétail, que celui qui l'a poussé paie pour chaque tête de bétail une obole. Que celui qui le désire dénonce auprès des mastroi celui qui commet un de ces délits. »

Pourquoi cet interdit ? Certains rituels impliquent la venue de nombreuses personnes, il faut donc les contrôler et les encadrer pour éviter les débordements. Il faut aussi éviter que les animaux ne souillent le sanctuaire de leurs déjections, ne se nourrissent de I'herbe et ne boivent I'eau du sanctuaire [120]. C'est pourquoi une amende est demandée en cas d'introduction d'animaux, puisque la divinité pourrait en être lésée. Le rejet de l'introduction des armes et objets métalliques [121] peut s'expliquer également par le souci d'éviter la violence et les débordements, tout comme l'introduction du vin dans le sanctuaire des divinités égyptiennes à Délos [122]. Un rituel est en outre un moment de trêve. Les conséquences de l'introduction de tels objets sont parfois précisées dans les règlements : une amende peut être prévue [123] et les objets étaient certainement consacrés.

L'eukosmia ne renvoie pas seulement au bon ordre et au calme lors des rituels, mais aussi à une certaine simplicité dans la tenue des participants. Les interdits portant sur les vêtements ou la parure des participants vont ainsi dans le sens de la limitation du luxe que l'on retrouve dans les lois somptuaires concernant les manifestations de deuil [124]. Les interdits ou les obligations vestimentaires pour les participants aux rituels pouvaient porter sur la couleur et la qualité des vêtements : on peut exiger des vêtements blancs [125] ou propres [126], non brodés [127] ou non colorés [128] ; la pourpre [129] est parfois interdite. On interdit aussi parfois les bijoux en or [130] ou on en limite le poids [131], de la même façon qu'on interdit les coiffures trop élaborées ou le maquillage [132] lors de certains rituels.

[124] LSAM 16/ CGRN 108, Gambreion, III ${ }^{\mathrm{e}}$ siècle av. J.-C. [125] LSAM 35/ CGRN 121 ; LSCG 68/ CGRN 126 ; LSS 59 ; LSAM 84 ; LSAM 14 ; LSCG 65/ CGRN 222.

[126] LSAM 6.

[127] LSS $32 ;$ LSCG 68/ CGRN $126 ;$ LSCG 94/ CGRN 173 ; LSAM 4 ; LSCG 65/ CGRN 222.

[128] LSS 33/ CGRN 127 ; SEG XXXVI 267 / LUPU 2005 n4; LSCG 65/ CGRN 222.

[129] Pouilloux, Rech I, n०155; LSS 33/ CGRN 127; LSCG 68/ CGRN 126; LSCG 65/ CGRN 222.

[130] LSCG 68/ CGRN 126; LSAM 6; LSAM 14.

[131] LSS 33/ CGRN 127.

[132] LSS 33/ CGRN 127 ; LSCG 68/ CGRN 126 ; LSS 91; LSAM 14 ; LSCG 65/ CGRN 222. 
Ces limitations du luxe sont présentées parfois clairement dans les règlements comme des indices de pureté, notamment lorsqu'on impose la couleur blanche ou la propreté des vêtements. Cette association est faite pour entrer dans un oikos sacré à Priène d'après une inscription du III ${ }^{\mathrm{e}}$ siècle av. J.-C. [133] ; on y précise qu'il faut « entrer dans le sanctuaire en

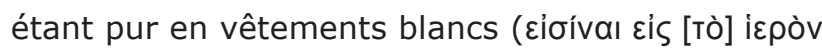
áyvòv $\dot{\varepsilon}[\mathrm{V}] \dot{\varepsilon} \sigma \theta \tilde{n} T ı \Lambda \varepsilon \cup \kappa[\tilde{n} ı]) \gg$.

Respecter ces interdits constitue en somme une forme de respect envers la divinité [134] ; c'est pourquoi la colère du dieu est envisagée dans certaines prescriptions. Il ne faut pas entrer dans le sanctuaire de Dionysos Bromios à Smyrne sans en respecter les règles « pour éviter de provoquer sa colère

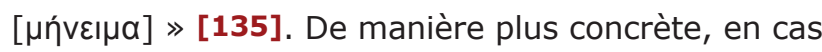
d'introduction d'objets ou de vêtements interdits, le contrevenant pouvait se risquer à une amende [136] et les objets et vêtements étaient consacrés à la divinité [137]. C'est ce qu'indique le règlement du III ${ }^{e}$ siècle av. J.-C. précisant les interdits lors des Mystères de Despoina à Lykosoura [138] :

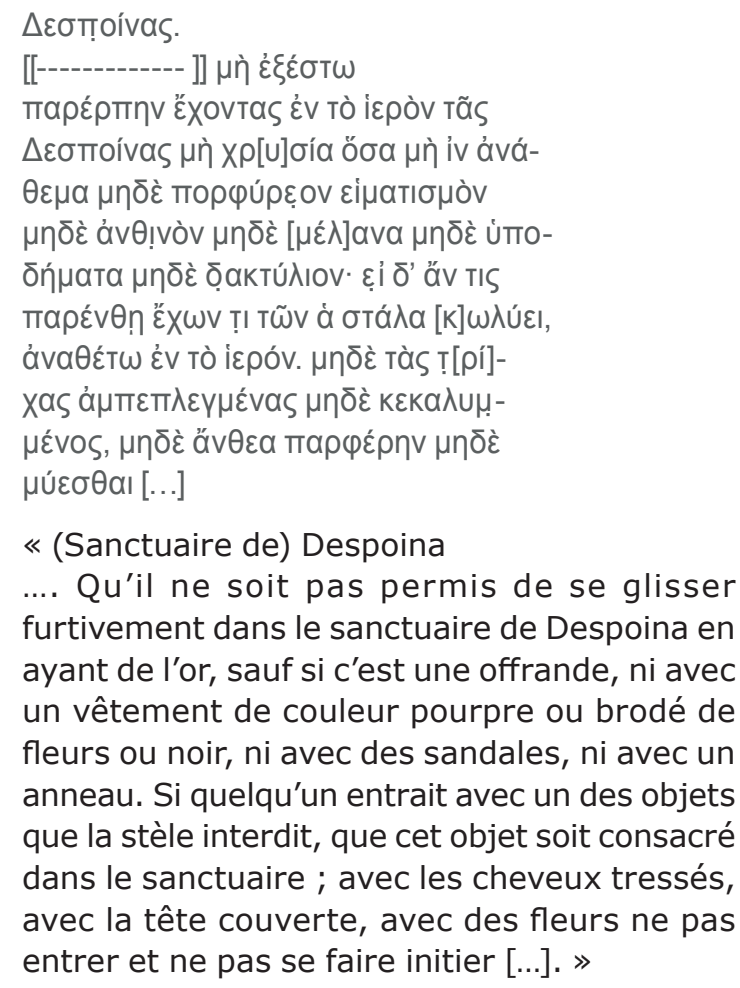

Le passage est intéressant dans la mesure où I'on constate que les objets interdits dans le sanctuaire deviennent la propriété de la déesse et sont de fait considérés comme un anathema, une offrande à la divinité. En somme, les objets ou vêtements luxueux sont réservés à la divinité et ne peuvent être qu'un anathema. Ainsi, contrairement aux pratiques interdites dans le sanctuaire, les objets interdits pouvaient-ils y laisser des traces matérielles, puisqu'on devait les trouver parmi les offrandes ou dans les sommes d'argent consacrées en cas d'amende.

\section{CONCLUSIONS}

Le corpus des prescriptions cathartiques, malgré son caractère elliptique, fournit finalement des indices variés des pratiques rituelles dans les sanctuaires du monde grec. Ces inscriptions affichées à l'entrée du sanctuaire marquent d'abord la limite entre deux espaces : I'espace sacré, l'espace du dieu, et l'espace extérieur, et les interdits qui y sont gravés précisent dans quelle mesure ceux qui veulent pénétrer dans le sanctuaire répondent aux règles de pureté requises uniquement dans le sanctuaire. Les individus, pour être purs, doivent se débarrasser des souillures laissées par des actions ou des situations qui font partie intégrante de la vie sociale. Les impuretés corporelles évoquées dans les règlements indiquent ainsi ce qui n'a pas cours dans le sanctuaire, et l'évocation des techniques de purification ou des instruments de lustration renvoient à des gestes rituels qui devaient être pratiqués avant d'honorer la divinité, quelle qu'en soit la manière. Les interdits portant sur des vêtements et des objets montrent quant à eux combien il est primordial de faire preuve d'eukosmia, de simplicité et de mesure face à la divinité pour ne pas risquer de la mettre en colère. Ils sont aussi des indices de la nature de certaines offrandes qui pouvaient être faites à la divinité, car les objets interdits qui étaient introduits dans le sanctuaire devenaient la propriété du dieu et se transformaient en anathemata.

[133] LSAM 35/ CGRN 121.

[134] La simplicité est de mise pour les participants : cf. Deshours 2006, p. 104.

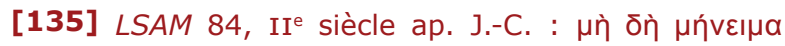
үÉvฑTaı. Voir aussi LSAM 6.

[136] LSS 32 ; LSCG 136/ CGRN 90.

[137] LSS 32 ; LSCG 65/ CGRN 222 ; LSCG 68/ CGRN 126.

[138] LSCG 68/ CGRN 126. 
Tableau $n^{\circ} 1$ : les interdits rituels dans les sanctuaires

\begin{tabular}{|c|c|c|c|c|c|c|c|c|}
\hline 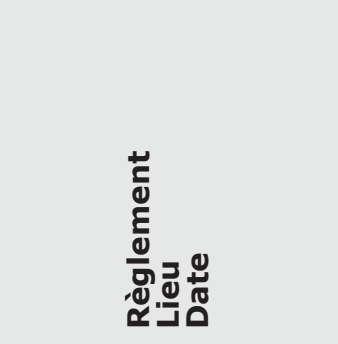 & 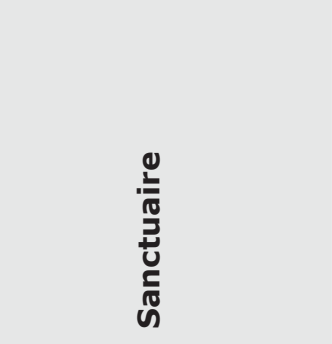 & 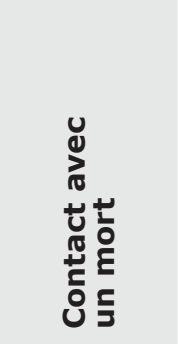 & 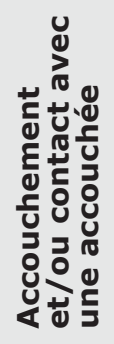 & 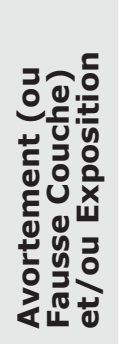 & 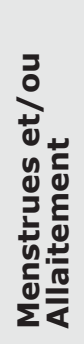 & 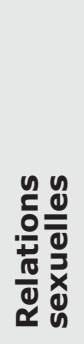 & 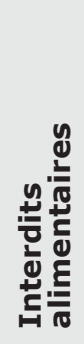 & 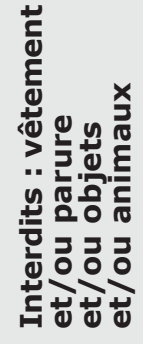 \\
\hline $\begin{array}{l}\text { LSCG 97/ CGRN } 35 \\
\text { Céos } \\
\text { ve siècle av. J.-C. }\end{array}$ & Règle les funérailles & $X$ & & & & $X$ & & \\
\hline $\begin{array}{l}\text { LSS 115/ CGRN } 99 \\
\text { Cyrène } \\
\text { IVe siècle av. J.-C. }\end{array}$ & Apollon & $x$ & $x$ & $X$ & & $x$ & & \\
\hline $\begin{array}{l}\text { LSAM 29/ CGRN } 71 \\
\text { Métropolis } \\
\text { Ionie } \\
\text { Ive siècle av. J.-C. }\end{array}$ & Déesse-Mère & $x$ & & & & $X$ & & \\
\hline $\begin{array}{l}\text { LSS 31/ CGRN } 65 \\
\text { Tégée, Arcadie } \\
\text { Ive siècle av. J.-C.. }\end{array}$ & Apollon & $\begin{array}{c}\text { Inscription } \\
\text { trop } \\
\text { abîmée }\end{array}$ & & & & & & \\
\hline $\begin{array}{l}\text { LSCG 68/ CGRN } 126 \\
\text { Lykosoura } \\
\text { Arcadie } \\
\text { III }^{\mathrm{e}} \text { siècle av. J.-C. }\end{array}$ & $\begin{array}{l}\text { Mystères de } \\
\text { Despoina }\end{array}$ & & $X$ & & $X$ & & & $X$ \\
\hline $\begin{array}{l}\text { LSCG 154/ CGRN } 148 \\
\text { Cos } \\
\text { III }^{\mathrm{e}} \text { siècle av. J.-C. }\end{array}$ & Déméter & $X$ & $x$ & $X$ & & & $X$ & \\
\hline $\begin{array}{l}\text { LSCG 156/ CGRN } 85 \\
\text { CoS } \\
\text { III }^{\mathrm{e}} \text { siècle av. J.-C. }\end{array}$ & $\begin{array}{l}\text { Zeus Polieus, Douze } \\
\text { dieux, Apollon Délios }\end{array}$ & $X$ & $X$ & $X$ & & & $x$ & \\
\hline $\begin{array}{l}\text { SEG XXVIII } 421 \\
\text { LUPU no 7/ CGRN } 155 \\
\text { Mégalopolis } \\
\text { Arcadie } \\
200 \text { av. J.-C. }\end{array}$ & $\begin{array}{l}\text { Isis, Sarapis et } \\
\text { Anoubis }\end{array}$ & & $X$ & $x$ & $X$ & $X$ & $x$ & \\
\hline $\begin{array}{l}\text { DECOURT \& } \\
\text { TZIAPHALIAS } 2015 \\
\text { Larissa } \\
\text { II }^{\mathrm{e}} \text { siècle av. J.-C. }\end{array}$ & $\begin{array}{l}\text { Artémis Phulakè, } \\
\text { Mên, Moire, Hélios, } \\
\text { Pan, Mogga, Alaia, } \\
\text { Lillaia, Adara, Lilla }\end{array}$ & & $X$ & $X$ & $X$ & $x$ & & $X$ \\
\hline $\begin{array}{l}\text { LSCG 171/ CGRN } 162 \\
\text { CoS } \\
\mathrm{II}^{\mathrm{e}} \text { siècle av. J.-C. }\end{array}$ & $\begin{array}{c}\text { Artémis et Zeus } \\
\text { Hikétios }\end{array}$ & & $X$ & $X$ & & $X$ & & \\
\hline $\begin{array}{l}\text { LSCG 124/ CGRN } 181 \\
\text { Erésos } \\
\text { Lesbos } \\
\mathrm{II}^{\mathrm{e}} \text { siècle av. J.-C. }\end{array}$ & $?$ & $X$ & $X$ & $X$ & & $x$ & & $X$ \\
\hline $\begin{array}{l}\text { LSS 54/ CGRN } 217 \\
\text { Délos } \\
\text { II }^{\mathrm{e}} \text { siècle av. J.-C. }\end{array}$ & $\begin{array}{l}\text { Dieux Syriens } \\
\text { (Fondation) }\end{array}$ & & $x$ & $X$ & $x$ & $x$ & $x$ & \\
\hline
\end{tabular}




\begin{tabular}{|c|c|c|c|c|c|c|c|c|}
\hline 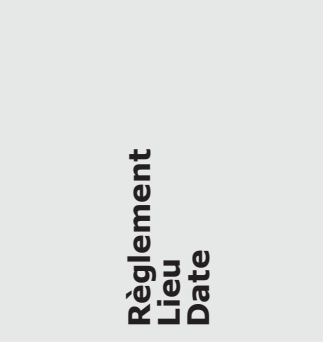 & 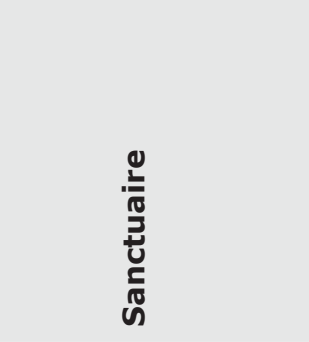 & 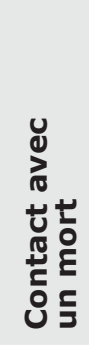 & 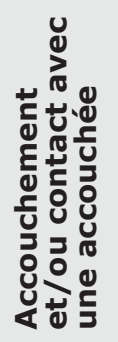 & 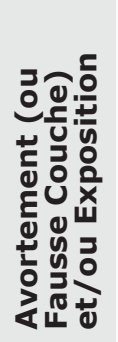 & 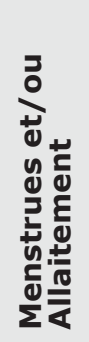 & 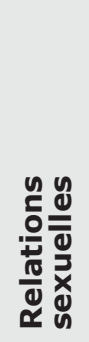 & 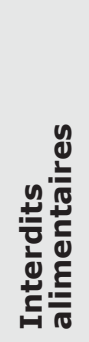 & 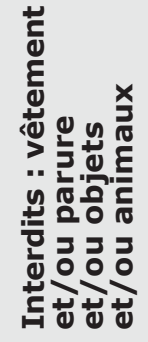 \\
\hline $\begin{array}{l}\text { LSCG 95/ CGRN } 203 \\
\text { Délos } 166 \text { av. J.-C. } \\
\text { Après } 16 \text {. }\end{array}$ & Artémis & & & & & $x$ & $\mathrm{x}$ & \\
\hline $\begin{array}{l}\text { LSAM 18/ CGRN } 211 \\
\text { Maeonie } \\
\text { Lydie } \\
147-146\end{array}$ & Cybèle & $\mathrm{x}$ & & & & $\mathrm{x}$ & & \\
\hline 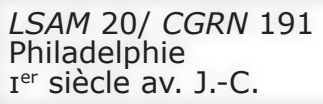 & Zeus Sôter & & & $\mathrm{x}$ & & $\mathrm{x}$ & & \\
\hline $\begin{array}{l}\text { LSAM 12/ CGRN } 212 \\
\text { Pergame } \\
\text { Apres } 133 \text { av. J.-C. }\end{array}$ & Athéna Nikèphoros & $\mathrm{x}$ & $\mathrm{x}$ & & & $x$ & & \\
\hline $\begin{array}{l}\text { LSS } 59 \\
\text { Délos } \\
116 / 115\end{array}$ & $\begin{array}{l}\text { Zeus Kynthios et } \\
\text { Athéna Kynthia }\end{array}$ & & & & & $x$ & $\mathrm{x}$ & $\mathrm{x}$ \\
\hline 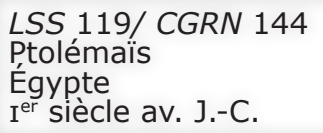 & $?$ & & $x$ & $\mathrm{x}$ & $\mathrm{x}$ & $\mathrm{x}$ & & $\mathrm{x}$ \\
\hline $\begin{array}{l}\text { LSCG 65/ CGRN } 222 \\
\text { Andanie } \\
92 \text { av. J.-C. }\end{array}$ & Déméter & & & & & & & $\mathrm{x}$ \\
\hline $\begin{array}{l}\text { LSAM 51/ CGRN } 214 \\
\text { Milet } \\
\text { Ir siècle ap. J.-C. }\end{array}$ & Artémis Kithônè & $\mathrm{x}$ & $\mathrm{x}$ & & & $x$ & & \\
\hline 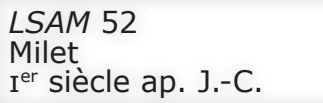 & Asclépios & & $\mathrm{x}$ & & & & & \\
\hline 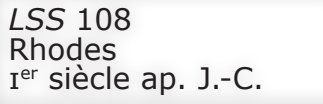 & $\begin{array}{l}\text { Sarapis? } \\
\text { Asclépios? }\end{array}$ & & & & & $\mathrm{x}$ & $\mathrm{x}$ & \\
\hline $\begin{array}{l}\text { LSAM } 84 \\
\text { Smyrne } \\
\text { II }^{\mathrm{e}} \text { siècle ap. J.-C. }\end{array}$ & Dionysos Bromios & $x$ & & $\mathrm{x}$ & & & $\mathrm{x}$ & $x$ \\
\hline $\begin{array}{l}\text { LSS } 106 \\
\text { Camiros } \\
\text { Rhodes }\end{array}$ & $?$ & $\mathrm{x}$ & & & & & & \\
\hline 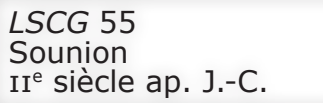 & Mên & $\mathrm{x}$ & & $x$ & $\mathrm{x}$ & $\mathrm{x}$ & $\mathrm{x}$ & \\
\hline $\begin{array}{l}\text { LSCG } 99 \\
\text { Céos } \\
\text { III }^{\mathrm{e}} \text { siècle ap. J.-C. }\end{array}$ & ? & & & $\mathrm{x}$ & & & & \\
\hline $\begin{array}{l}\text { LSS } 91 \\
\text { Lindos } \\
\text { Rhodes } \\
\text { III }{ }^{\mathrm{e}} \text { siècle ap. J.-C }\end{array}$ & Athéna & $\mathrm{x}$ & $\mathrm{x}$ & $\mathrm{x}$ & & $\mathrm{x}$ & & $\mathrm{x}$ \\
\hline $\begin{array}{l}\text { LSAM } 14 \\
\text { Pergame } \\
\text { III }{ }^{\mathrm{e}} \text { siècle ap. J.-C }\end{array}$ & Asclépios & $\mathrm{x}$ & $x$ & & & & & $\mathrm{x}$ \\
\hline
\end{tabular}


Tableau $\mathrm{n}^{\circ} 2$ : animaux, vêtements et objets interdits

\begin{tabular}{|c|c|c|c|c|c|c|c|c|c|}
\hline 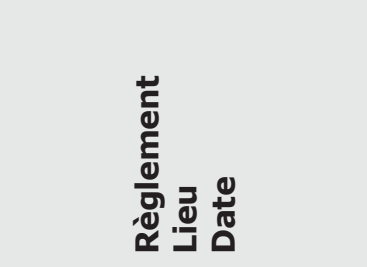 & 옹 & 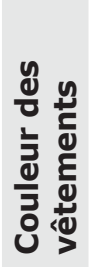 & 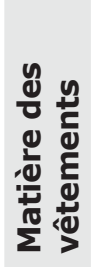 & 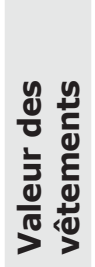 & 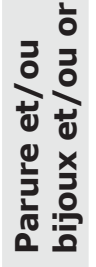 & $\begin{array}{l}y \\
\frac{y}{0} \\
\frac{0}{0} \\
0 \\
y \\
0 \\
\frac{1}{3} \\
\frac{7}{4}\end{array}$ & 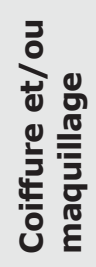 & $\frac{x}{\sqrt{0}}$ & 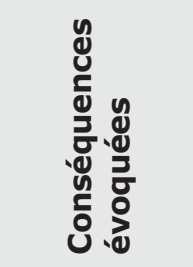 \\
\hline 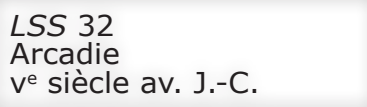 & Déméter & & & $x$ & & & & & $\begin{array}{c}\text { Objets } \\
\text { consacrés } \\
\text { ou amende }\end{array}$ \\
\hline $\begin{array}{l}\text { LSS 63/ CGRN 27, } \\
\text { Thasos } \\
440 \text { av. J.-C. }\end{array}$ & Héraclès Thasien & & & & & & & $\begin{array}{c}\mathrm{X} \\
\text { (sacrifice) }\end{array}$ & \\
\hline $\begin{array}{l}\text { Pouilloux, Rech I, nº155 } \\
\text { Thasos } \\
\text { Iv }{ }^{\mathrm{e}} \text { siècle av. J.-C. }\end{array}$ & $?$ & & & $x$ & & & & & \\
\hline $\begin{array}{l}\text { LSCG 136/ CGRN } 90 \\
\text { Ialysos } \\
300 \text { av. J.-C. }\end{array}$ & Alectronè & & $\mathrm{X}$ & & & & & $x$ & $\begin{array}{l}\text {-amende } \\
\text {-purification } \\
\text {-sacrifice } \\
\text {-impiété }\end{array}$ \\
\hline $\begin{array}{l}\text { LSAM 35/ CGRN } 121 \\
\text { Priène } \\
\text { III }^{\mathrm{e}} \text { siècle av. J.-C. }\end{array}$ & $\begin{array}{l}\text { Entrée dans un } \\
\text { oikos sacré }\end{array}$ & $X$ & & & & & & & \\
\hline $\begin{array}{l}\text { LSS 33/ CGRN } 127 \\
\text { Patras } \\
\text { III I }^{\mathrm{e}} \text { siècle av. J.-C. }\end{array}$ & Fêtes de Déméter & $x$ & & $\mathrm{X}$ & $x$ & $x$ & $\mathrm{X}$ & & $\begin{array}{l}\text {-purification } \\
\text {-sacrilège }\end{array}$ \\
\hline $\begin{array}{l}\text { LSCG 68/ CGRN } 126 \\
\text { Lykosoura } \\
\text { III }^{\mathrm{e}} \text { siècle av. J.-C. }\end{array}$ & $\begin{array}{l}\text { Mystères de } \\
\text { Despoina }\end{array}$ & $x$ & $x$ & $x$ & $x$ & $x$ & $x$ & $x$ & $\begin{array}{l}\text { Objets } \\
\text { consacrés }\end{array}$ \\
\hline $\begin{array}{l}\text { Decourt § Tziaphalias, } \\
2015 \\
\text { Larissa } \\
\mathrm{II}^{\mathrm{e}} \text { siècle av. J.-C. }\end{array}$ & $\begin{array}{l}\text { Artémis Phylakè, } \\
\text { Mên, Moire, Hélios, } \\
\text { Pan, Mogga, Alaia, } \\
\text { Lillaia, Adara, Lilla }\end{array}$ & & & & & & & $x$ & \\
\hline $\begin{array}{l}\text { LSCG 124/ CGRN } 181 \\
\text { Erésos } \\
\text { II }^{\mathrm{e}} \text { siècle av. J.-C. }\end{array}$ & $?$ & & $\mathrm{X}$ & & & & & & \\
\hline $\begin{array}{l}\text { LSS } 56 \\
\text { Délos } \\
\text { II }{ }^{\mathrm{e}} \text { siècle av. J.-C. }\end{array}$ & $\begin{array}{l}\text { Divinités } \\
\text { égyptiennes }\end{array}$ & & $X$ & & & & & & \\
\hline $\begin{array}{l}\text { LSCG 94/ CGRN } 173 \\
\text { Délos } \\
\text { II }{ }^{\mathrm{e}} \text { siècle av. J.-C. }\end{array}$ & $\begin{array}{l}\text { Divinités } \\
\text { égyptiennes }\end{array}$ & & & $x$ & & $\mathrm{X}$ & & & \\
\hline $\begin{array}{l}\text { LSAM } 6 \\
\text { Chios } \\
\text { I }^{\text {er siècle av. J.-C. }}\end{array}$ & $\begin{array}{l}\text { Culte féminin } \\
\text { (Déméter ?) }\end{array}$ & $x$ & $x$ & & $\mathrm{X}$ & & & & $\begin{array}{l}\text { Colère de } \\
\text { la déesse }\end{array}$ \\
\hline $\begin{array}{l}\text { SEG XXXVI } 267 \\
\text { LUPU n } 4 \\
\text { Marathon } \\
61 / 60 \text { av. J.-C. }\end{array}$ & Pan & $x$ & & & & & & & \\
\hline $\begin{array}{l}\text { LSS } 59 \\
\text { D̦élos } \\
\text { Epoque romaine }\end{array}$ & $\begin{array}{l}\text { Zeus Kythios et } \\
\text { Athéna Kynthia }\end{array}$ & $x$ & $x$ & & & $x$ & & & \\
\hline $\begin{array}{l}\text { LSAM } 84 \\
\text { Smyrne } \\
\text { II }^{\mathrm{e}} \text { siècle ap. J.-C. }\end{array}$ & Dionysos Bromios & $x$ & & & & & & & $\begin{array}{l}\text { Colère du } \\
\text { dieu }\end{array}$ \\
\hline $\begin{array}{l}\text { LSS } 91 \\
\text { Lindos } \\
\text { Rhodes } \\
\text { III I siècle ap. J.-C. }\end{array}$ & $?$ & $x$ & $\mathrm{X}$ & & & & $X$ & & \\
\hline $\begin{array}{l}\text { LSAM } 14 \\
\text { Pergame } \\
\text { III }{ }^{\mathrm{e}} \text { siècle ap. J.-C. }\end{array}$ & $\begin{array}{l}\text { Asclépios } \\
\text {-incubations }\end{array}$ & $x$ & $x$ & & $X$ & & $X$ & & \\
\hline $\begin{array}{l}\text { LSAM } 77 \\
\text { Tlôs } \\
\text { ? }\end{array}$ & $?$ & & & $x$ & & $x$ & & & Amende \\
\hline
\end{tabular}




\section{REMERCIEMENTS}

Je tiens à remercier vivement Anne Jacquemin pour ses conseils lors de la rédaction de cet article.

\section{BIBLIOGRAPHIE}

Augrer, Marie, 2015, « Le corps et ses souillures dans les pratiques religieuses en Grèce ancienne », dans Philippe Guisard \& Christelle Laizé (dir.), Le corps, Paris (Collection Cultures Antiques), p. 570-598.

Augrer, Marie, 2016, «La souillure et le genre dans le monde méditerranéen antique », Ktèma. Civilisation de l'Orient, de la Grèce et de Rome antiques 41, p. 279-318.

Augrer, Marie, 2017a, « Nul n'entre ici s'il n'est purifié. Corps, gestes et souillure dans les prescriptions cathartiques de la période archaïque à la période romaine. », OTIUM Archeologia e Cultura Antica del Mondo Antico 2, Article 18.

Augrer, Marie, 2017b, «Le sang menstruel dans les prescriptions cathartiques », dans Lydie Bodiou \& Véronique Mehl (dir.) L'Antiquité écarlate. Le sang des Anciens, Rennes, p. 95-111.

Bendlin, Andreas, 2007, «Purity and Pollution», dans Daniel Ogden (éd.), A Companion to Greek Religion, Oxford, p. $178-189$.

Betegh, Gabor, 2014, dans Carl A. Huffmann (éd.), A History of Pythagoreanism, Cambridge, p. 149-166.

BOEHRINGer, Sandra, 2018, «La sexualité dans les sociétés grecque et romaine », dans Sylvie Steinberg (dir.), Une histoire des sexualités, Paris, p. 15-91.

Bodrou, Lydie \& Meht, Véronique, 2019, Dictionnaire du corps dans l'Antiquité, Rennes.

Bodrou, Lydie, 2011, « Les inquiétantes humeurs féminines. L'exemple du lait maternel dans les écrits médicaux de l'époque classique », La femme, la parenté et le politique : parcours sensible d'une historienne, mélanges en l'honneur de Claudine Leduc, Pallas 85.

Bodrou, Lydie \& BRULÉ, Pierre, 2011, « Naître en Grèce ancienne. Des accouchements pour le moins mouvementés », dans Lydie Bodiou, Véronique Mehl \& Myriam Soria (éd.), Corps ravagés, corps outragés, Regards croisés de l'Antiquité au Moyen Âge, Turhout.

DecouRT, Jean-Claude \& Tziaphallias, A., 2015, « Un règlement religieux de la région de Larissa : cultes grecs et « orientaux 》», KERNOS 28, p. 13-51.

DeshouRs, Nadine, 2006, Les mystères d'Andania, Étude d'épigraphie et d'histoire religieuse, Paris.

Detienne, Marcel \& Vernant, Jean-Pierre, 1979, La cuisine du sacrifice en pays grec, Paris.

Dobias Lalou, Catherine, 1988, Recherches sur le dialecte des inscriptions grecques de Cyrène, Lille.

DucAT, Jean, 1964, « Périrrhantèria », Bulletin de correspondance hellénique 88/2, p. 577-606.

ECK, Bernard, 2012, La Mort rouge, Homicide, guerre et souillure en Grèce ancienne, Paris.

EIDINow, Esther, 2011, «Sex, Religion, and the Law », dans Mark Golden \& Peter Toohey (éd.), A Cultural History of Sexuality, in the Classical Word, Oxford - New York, p. 87-106.

FrISONE, Flavia, 2000, Leggi e regolamenti funerari nel mondo greco I. Le fonti epigrafiche, Galatina.

GARNSEY, Peter, 1999, Food and Society in Classical Antiquity, Cambridge.

GRAND-CLÉment, Adeline, 2018, « «Il est interdit de...». Rituels et procédures de régulation sensorielle dans le monde grec ancien: quelques pistes de réflexion », Mythos. Rivista di Storia delle Religioni. 12, p. 49-68.

LuPu, Eran, 2005, Greek Sacred Law, A Collection of New Documents, Leiden - Boston.

MeHL, Véronique, 2009, « Le temps venu de la maternité », dans Lydie Bodiou \& Véronique Mehl (éd.), La religion des femmes en Grèce ancienne, Mythes, Cultes et Sociétés, Rennes, p. 193-206.

MILLS, Harrianne, 1984, « Greek Clothing Regulations: Sacred and Profane? », Zeitschrift für Papyrologie und Epigraphik 55, p. 255-265.

Moulinier, Louis, 1950, Le pur et l'impur dans la pensée et la sensibilité des Grecs jusqu'à la fin du IVème siècle av. J.-C., Paris.

Osborne, Catherine, 1995, «Ancient Vegetarianism », dans John Wilkins, David Harvey \& Mike Dobson (éd), Food in Antiquity, Exeter, p. 214-224.

PARKer, Robert, 1983, Miasma, Pollution and Purification in early Greek Religion (réédité en 1996), Oxford.

PrMPL, Heidrun, 1997, Perirrhanteria und Louteria: Entwicklung und Verwendung grosser Marmor- und Kalksteinbecken auf figürlichem und säulenartigem Untersatz in Griechenland, Berlin.

SokolowsKI, Franciszek, 1955, Lois sacrées de l'Asie Mineure, Paris.

SokolowsKI, Franciszek, 1962, Lois sacrées des cités grecques, supplément, Paris.

SoKolowsKI, Franciszek, 1969, Lois sacrées des cités grecques, Paris.

WÄCHTER, Theodor, 1910, Reinheitsvorschriften im grieschischen Kult, Giessen.

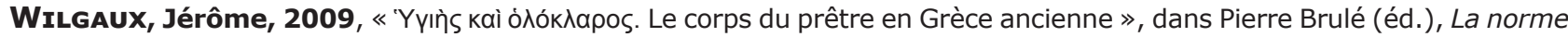
en matière religieuse en Grèce ancienne, Actes du XI colloque du CIERGA, Rennes, septembre 2007, Liège, p. 231-242. 